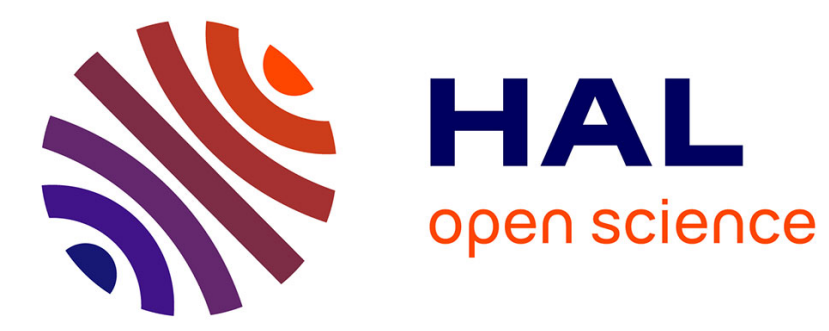

\title{
La villa gallo-romaine des Prés-Bas, à Loupian (Hérault)
} Henri Lavagne, Richard Prudhomme, Daniel Rouquette

\section{To cite this version:}

Henri Lavagne, Richard Prudhomme, Daniel Rouquette. La villa gallo-romaine des Prés-Bas, à Loupian (Hérault). Gallia - Fouilles et monuments archéologiques en France métropolitaine, 1976, 34 (1), pp.215-235. 10.3406/galia.1976.1550 . hal-01938488

\section{HAL Id: hal-01938488 \\ https://hal.science/hal-01938488}

Submitted on 11 Mar 2020

HAL is a multi-disciplinary open access archive for the deposit and dissemination of scientific research documents, whether they are published or not. The documents may come from teaching and research institutions in France or abroad, or from public or private research centers.
L'archive ouverte pluridisciplinaire HAL, est destinée au dépôt et à la diffusion de documents scientifiques de niveau recherche, publiés ou non, émanant des établissements d'enseignement et de recherche français ou étrangers, des laboratoires publics ou privés.

\section{(이)(\$)}

Distributed under a Creative Commons Attribution - NonCommercial - NoDerivatives| 4.0 


\title{
LA VILLA GALLO-ROMAINE DES PRÈS-BAS A LOUPIAN (Hérault)
}

\author{
par Henri LAVAGNE, Richard PRUDHOMME ef Daniel ROUQUETTE
}

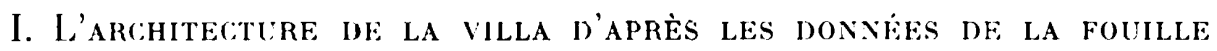

C'est une courte note parue en 1930 dans la Revue des Musées ${ }^{1}$ qui nous a permis de retrouver ce site mis au jour fortuitement à cette époque au cours de travaux agricoles. Après quelques prospections de surface effectuées fin 1962, nous avons pratiqué l'année suivante des sondages dans une parcelle inculte, entre les vignes. Au cours de l'un de ces sondages, l'abbé E. Maistre rencontra une mosaïque à $0,60 \mathrm{~m}$ de profondeur. Une pièce complète avec ses deux seuils en place fut ainsi dégagée (pièce B, fig. 1). M. H. Gallet de Santerre, alors Directeur des antiquités historiques de la région Languedoc-Roussillon, fit poursuivre les recherches qui révélèrent la présence d'une nouvelle mosaïque. Grâce à l'aide du Maire de Loupian et du Conseil général du département, l'acquisition de la parcelle (un peu plus d'un hectare) par la commune de Loupian fut réalisée en 1967. Enfin, en 1970, sur proposition de M. G. Barruol, Directeur des antiquités historiques, le site a été classé et un hangar fermé a été conçu pour abriter les pavements AA', B et C'2.

1 Revue des .Musées, 26, 1930, p. 168: "au cours d'un travail dans une vigne appartenant a 11. A. Bécole, les ouvriers ont mis au jour des fragments de mosaigque fort belle et très bien conservée. bes pièces completes ont eté également retrouvées. Il est hors de doute qu'il y a eu sur cet emplacement à l'époque gallo-romaine une riche villa patricienne ". Cette information a été reprise avec une erreur sur le lieu-dit par E. Boxxet, Forma Héraull, X, Paris, 1946, p. 17, no 43. Les diverses étapes des fouilles ont été notées dans les Informalions de la Circonscription du Languedoc-Koussillon : cf. Gallia, 22, 1964, p. 493, fig. 22 l'atside A ;27, 1969, p. $395 ; 31$, 1973, p. 494. fig. 17 detail de la mosaíque de la piece $\mathbf{E}$.

2 Cous profitons de cette premiere publication pour renouveler notre gratitude à tous ceux qui ont permis le sauvetage de ce bel ensemble. En premier lieu, .M.M. R. Pissère et H. Gallet de Santerre, respectivement Prifet de l'Hérault et Directeur des antiquités historiques à l'époque de la découverte, qui ont assuré le classement provisoire du gisement archéologique. Le Conseil génèral du département, et particulièrement .I. A. Montel, élu cantonal, M. .I. Courrieu, Maire de Loupian, tous deux décédes depuis. MII. J. Armengaud et G. Benezech, l'ancien et l'acluel Maire de Loupian, qui nous ont souvent facilité la tàche, M. Gr. Barruol, Directeur des antiquilés historiquers qui a pu réaliser la protection des premieres mosiąques. Enfin, nous ne saurions oublier tous les amis et chercheurs régionaux qui nous ont aidés et encouragés, en particulier : MM. Ie chanoine Giry, labbe E. Maistre, J. Belol, L. Vatte, J.-P. Pappalardo, R. Julie, II. Sutherland, I). Espinasse, les Membres de la Société d'Études scientifiques de Sète, du Centre de recherches archéologiques des Chênes-verts de Montpellier, du Groupe de recherche et d'études clermontais, ainsi que les photographes de l'Inventaire régional, MM. M. Jescossy ef J. Vallon à qui sont dues toutes les photo. graphies de cet article. 

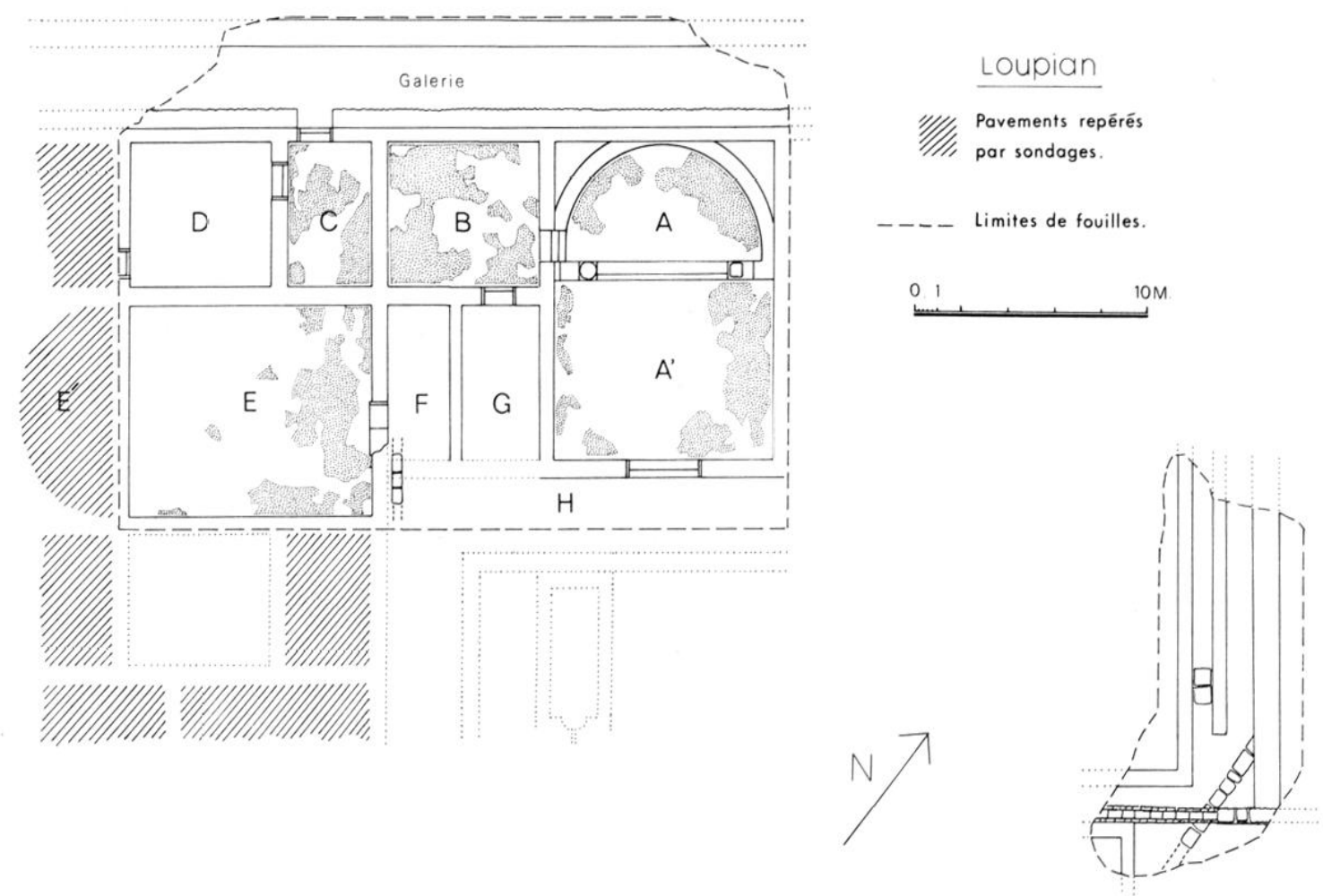

I Plan de la villa, Etal des fouilles en 1975.

Cé n'est ni la toponymie ni la tradition orale qui ont été à l'origine de la découverte ${ }^{3}$. La dénomination du lieu-dit les Prés-Bas s'explique par la situation de ce tènement. i deux kilomètres de Meze, ces terres ne sont qu'à quelques mètres au-rlessus des eaux du Bassin de Thau", dont le rivage actuel est à $800 \mathrm{~m}$ au sud de l'implantation gallo-romaine. Il est probable que dans l'Antiquité, cette dépression, drainée par le ruisseau du Bourbou, présentait un aspect marécageux. Ln terrain plus sain a donc été choisi pour l'établissement de la villa, à savoir les premières terrasses formant la bordure ouest de ce bas-fond̃. De cet endroit, la vue embrasse une bonne partie de l'étang de Thau ainsi que la silhouette de la montagne de Site ${ }^{6}$ qui sépare celui-ci de la Méditerranée. Située à mi-distance des stations routières de Cessero (Saint-Thibéry) et Forum Domilii (Montbazin), à $2 \mathrm{~km}$ au surl de la mansio de Frontiana a a point où la voie Jomitienne

3 R. Cinsalan, Problemalique de la villa gallo-rominine, dans La villa romana, Giornala di Studi-Russi, Florence 1971, dans Sludi Romagnoli, 1971, p. 39, 41. On n:" salurail relenir la forme "Lupianum villa "mentionnée dans la

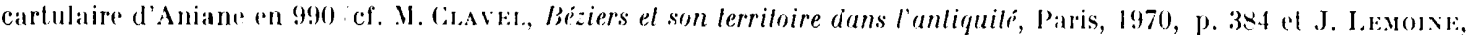
Toponymie du Languedoc et de la Gascogne, Paris, 1975, p. I2X at 146. La suite du frxle "castro cum manso podio" montre bien qual ne sagil pas de la villa mais du village el de son chateau siturs a 900 m plus au nord.

1 Festus Avienus, Ora marilima, .607 . Taurum paludem...

5) Parcelles communales E, $n^{\circ} 69$ a 74 . Coordomnés au centere du sile : $x=703,40 ; y=127,60 ;$ carte JGX, Sille $1^{\circ} 5$, XXVII, 14.

6 Fistus Avienus, ibid., 1.604 , Selins mons...

7 Cous venons de retrouver l'emplacement de celte station mentionnese sur le qual rieme vase de Vicarello.

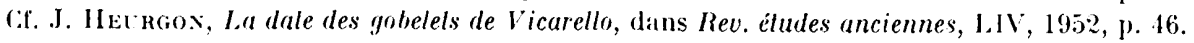


est la plus proche du littoral, la villa était facilement arcessible par un diverticule partant de cette grande artere. Cne de res voies secondaires rontinuait d'ailleurs jusqualu rivage, au lieu-rlit Port-de-Loupian où existe un autre établissement gallo-romain à fonction vraisemblablement maritime. L'une agricole. l'autre commerciale, les deux exploitations proches et complémentaires, appartenaient peut-être à un même propriétaire.

Insuffisamment avancés, les travaux ne permettent pas une description définitive de l'implantation antique et la fonction des diverses pièces de l'edifice ne pourra ètre précisé que par la suite des fouilles. Artuellement. une quinzaine de pièces a eté reconnue par sondages, dont douze portent des parements an mosaïque. Malgré les incertitudes qui demeurent, nous avons pensé qu'il etait utile de faire connaitre ces structures et d'en dresser un plan préliminaire (fig. 1).

Clölure el galerie nord : les diverses salles . I. B. C. I) situées au nord de la rilla sont appuyées: sur un tris gros mur, large de 1.15 m, dont la construction présente deux techniques différentes. Contre sa face interne. batie en moellons irréguliers de calcaire coquillier, a élé coulé un fort béton - au sens moderne du mol constitué de gros éclats de calcaire jurasique trés durs noyés dans un mortier de chaux. Valgré l'ignorance où nous sommes de la hauteur que pouvait alteindre ce mur, nous pouvons penser que ce doublement extérieur constituait une protection thermique contre les vents froids d'hiver soufllant du nord. Parallible a ce dernier et a 2.50 m de lui, court un second mur. large de prese d'un m, bati en moellons réguliers de moyen appareil. Plusieurs sondages à l'extérieur de ce mur n'ont rencontré aucun lémoignage archéologique et renforcent l'hypothèse selon laquelle nous devons y voir un mur de clóture pour la zone nord de la rilla. Il apparail ainsi que ces structures limitent une galerie extérieure aux batiments. La partie dégagée a révélé un sol de lerre ballue.

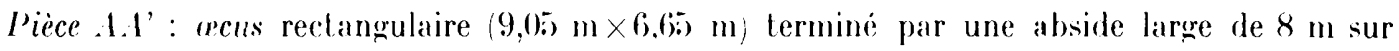
5) mil de rayon, dont le sol mosaïqué est surélevé de $10^{\mathrm{m}} \mathrm{m}^{8}$. Outre la mosiäque polychrome qui orne le sol, on note des restes de plinthe en marbre blanc épousant la courbure de la partie absidée. Cette abside a été inscrite dans une salle rectangulaire antérieure, dont les restes de pavement en terrazzo- signinum sont encore visibles. De plus. le mur séparant, à l'origine, celte pirce de la salle reclangulaire 1 ', étail décoré sur ses deux fares de plinthes en marbre blanc, et porlait a ses deux extrémités deux colonnes en calcaire fin dont on apercoit les bases. Lors des modifications qui ont aflecté la villa au Bas-Limpire, le mur a été arasé au niveau du sol portant la mosaique $A$, formant ainsi la marche existant entre les deux pieces. Enfin, dans un dernier slade d'occupation, les sols de mosaïques $\mathrm{A}$ et $\mathrm{A}$ ', ont été recouverts d'une chape en béton de tuileau. Ce revètement grossier s'enlève facilement par plaques lorsque le pavement. en mosaïque qui lui sert d'assise existe encore en dessous. Mallheureusement l'opus lessellatum devait avoir ... en partie -- disparu au moment où le niveau fut ainsi rechargé, car le plus souvent le décapage n'a pas révélé de mosaíque. Dans la pièce A'. nous verrions volontiers un des acus de la demeure; comme il est fréquent, il communique par une large ouverture (3 m) avec un péristyle partiellement dégagé. sur les longs cólés, des plinthes de marbre hlanc sont conservées, ainsi qu'un lambris de marbre mouluré sur le cóté est.

Piece $B$ : de forme quadrangulaire $(6,40 \mathrm{~m} \times 5,60 \mathrm{~m})$, elle posisde deux seuils en place ouvrant sur les pièces 1 et (i. Dans l'angle nord-est, un creusement. pratiqué vraisemblablement dans l'antiquité, affecte la mosaique el son support. De forme bi-elliptique, cette sorte de bassin laisse

8 Des dispositions semblables ont ele releveres sur les phans de maisons antiques dudipes par R. ReBtrFat,

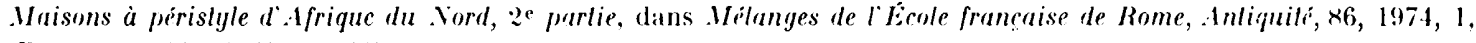
Bamasa, p. 448, Colta, p. 449. 
apparaître à un niveau inférieur un pavement en terrazzo-signinum comme dans la pièce $\Lambda$, orné d'un décor de croisettes noires. sur ce sol est placée une mosiäque polychrome à canevas géométrique.

l'ièce $\mathrm{C}$ : rectangulaire (3.90 $\mathrm{m} \times 5.65)$. elle a deux seuils en place. l'un ourrant sur la pièce 1 ), l'autre sur la galerie extérieure nord. Au sol. une mosaïque géométrique polychrome. Ce pavement recouvre un sol antérieur en bèton rose. I'ne fracture dans la mosaïque a permis de constater que sur le béton. on a plaré une couche de fragments d'enduits. peints en rouge. qui servent de lit il la mosiäque.

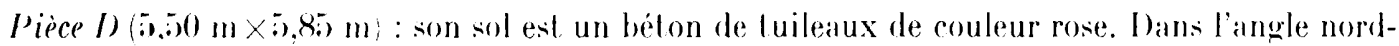
ouest, a la base du gros mur du fond, on remarque quelques laces de peinture jaune. lne fissure en demi-cercle affecte le sol de la piéce el semble récéler l'existence d'un sol antérieur en forme d'abside. Jans l'angle du mur ouest, un seuil en place ouvre sur une autre pièce.

P'ièce $E$ : c'est la plus grande des pièces dégagées jusqu'à ce jour $(10,45) \mathrm{m} \times 8.85$ ) m). Elle communique. par une banquette légèrement surélevée recouverte d'un béton fin, aver une abside. comme pour les pièces . I.'. Ce mème agencement peut aroir eu les mòmes fonctions. seul le seuil dénote une ouverture moins large. Le sol est recouvert d'une mosiäque polychrome. Plinthes de marbre conservées sur ?3 còtés.

P'ièce E' : abside complémentaire de la satle E. elle mesure $8.40 \mathrm{~m}$ sur un rayon de $4.50 \mathrm{~m}$. sol en mosiäque à décor géométrique polychrome.

Pièce $F$ : le sol en béton est situé à un niveau plus bas que celui de toutes les autres pièces et peut avoir constitué le support d'un hypocauste (:, $\$ 0 \times 2,70)$. Bien qu'aucun élément de la suspensura n'ait été rencontré dans la fouille. quelques briques carrées pouvant appartenir à des pilettes ont été trouvées en remplissige.

P'ièce ( $;$ : de mème lonqueur que la précédente, mais un peu plus large (33.25 mi; nous y voyons plutòt une cour ou un passage non couvert donnant acces a la piece B.

Autour de cel ensemble, cinq autres pieces ont été individualisées par sondages; elles comportent toules des mosaïques qui seront éludiées par la suite.

Pièce II : c'est une galerie au sol de béton rose dont deux cootés formant angle droil ont été partiellement dérugués. Un bassin non fouillé, situé au sud de l'aile est-ouest de re passage, nous renforce dans l'idée qu'il s'agit des deux còtés du péristyle, autour duquel s'ordonnent et prennent jour les diverses pières de li villa.

Cous sommes done en présence d'une grande villa. orrupée vraisemblablement des

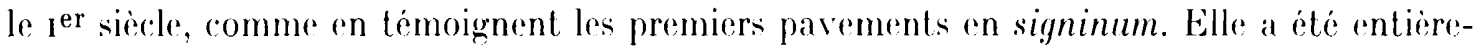
ment remanièe et luxueusement décorée à l'époque tardive à an juger par l'ensemble des mosaïques de bonne qualité qui ornent le dernier niveau d'habitation. Les premiers dégagements ne nous ont fourni aucun indice sùr de datation, et aucun materiel pouvant aider à préciser la chronologie n'a éte trouvé. En attendant la dépose de la mosaïque $\mathrm{E}$, qui donnera peut-être les éléments d'une stratigraphie, il faut s'en remettre à l'analyse stylistique des motifs des mosiäques.

Daniel Rorgutetr. 


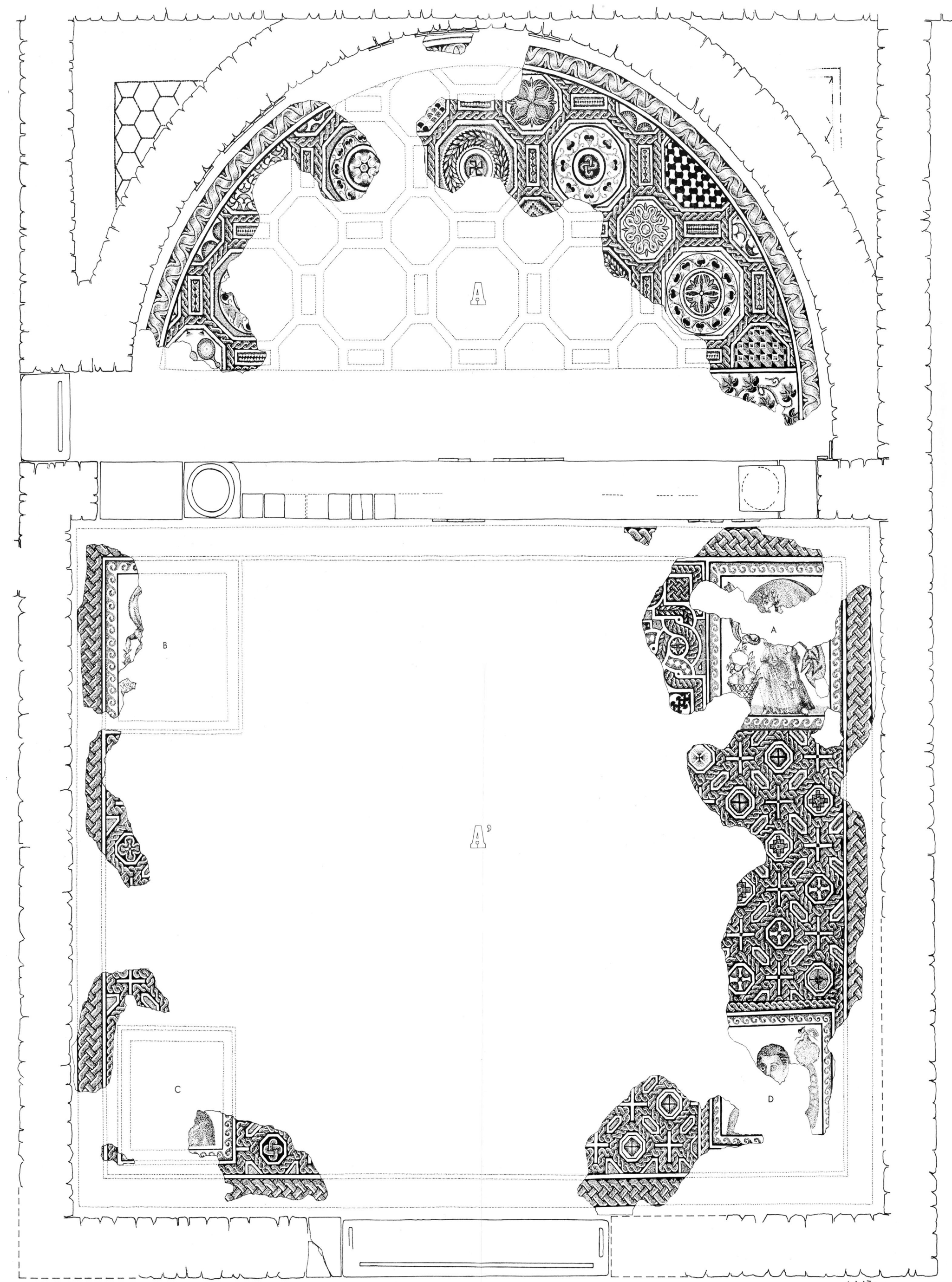





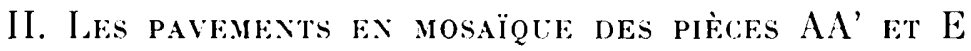

L'étude des douze pavements en mosaïque reconnus la plupart par sondages, et entièrement mis au jour pour quatre d'entre eux, ne saurait entrer dans les limites d'un seul article. Aussi avons-nous dù nous limiter ici aux mosäqques de deux pièces. Nous avons choisi les pavements des salles AA' et E pour les traits communs qu'ils partagent: ils constituent l'ornement de pièces de grandes dimensions qui devaient être des salles d'apparat, leurs qualités d'exécution sont sensiblement égales, les rappels de motifs et les analogies de style amènent à les regrouper en un ensemble relativement cohérent malgré des différences de facture, et nous pensons qu'il convient de les placer à la même époque.

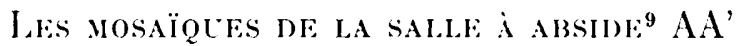

L'histoire complexe des modifications architecturales des deux parties de la pièce AA', telles qu'on peut les déduire des observations tirées des fouilles elles-mêmes, ne nous donne pas d'éléments certains pour décider de la chronologie absolue de leurs mosaïques; mais pour établir une chronologie relative, il est clair que l'on doit distinguer d'abord un sol appartenant au premier état d'occupation du sol de la villa, dont deux fragments sont conservés. Le premier est repérable sur une petite surface $(80 \mathrm{~cm} \times 60 \mathrm{~cm})$ dans l'écoinçon nord-est de l'abside, à une profondeur de $80 \mathrm{~cm}$ au-dessous du niveau de la mosaïque formant le pavement actuellement visible. C'est un terrazzo-signinum ${ }^{10}$ assez grossier, à fond blanc grisàtre, sur lequel apparait l'angle d'un tapis en opus lessellalum où des tesselles noires dessinent un réticulé losangé. Presque symétrique de ce premier sol, par rapport à l'axe de l'abside, se trouve l'angle d'un autre pavement, dans l'écoinçon nord-ouest, qui comporte un décor du même genre : lerrazzo-signinum portant en cubes noirs un canevas d'hexagones réguliers tangents. Ces deux types de pavements qui apparaissent en Italie dès la fin du ${ }_{\mathrm{I}}{ }^{\mathrm{e}}$ siècle arant notre ère sont attestés dans le Midi de la Gaule depuis le milieu du dernier siècle avant J.-C.. ${ }^{11}$. Le mélange des deux techniques (opus signinum et opus lessellalum), la simplicité des schémas et les quelques exemples rassemblés dans les cinq premiers volumes du Recueil général des mosaïques de la Gaule nous invitent à placer ces deux sols entre la fin du ${ }^{\text {er }}$ siècle av. J.-C. et le milieu du $\mathbf{I}^{\mathrm{er}}$ ap. J.-C. Ils témoignent donc de l'ancienneté de l'habitation, qui devait offrir une ou deux pièces carrées à l'emplacement de la partie absidée qui occupe aujourd'hui le niveau supérieur. Des mosaïques de la seconde période, un quart à peine est encore visible, tant dans l'abside A que dans l'œecus A' qui la prolonge. Un simple coup d'œil au relevé des deux pavements (fig. 2) montre que malgré des différences très réelles dans le détail, l'unité de la

9 L'abside est ici la partie an demi-cercle qui prolonge la partie rectangulaire de la piece $A$ '.

10 Sur ce terme el son emploi, cf. H. Strkx, Recueil général des mosaïques de la liaule, I, 3, p. 11, note 8.

11 H. Rollaxd, Observations sur les mosaïques de Gianum, dans Archivo espanol di arqueologia, 1952, p. 8-14. 


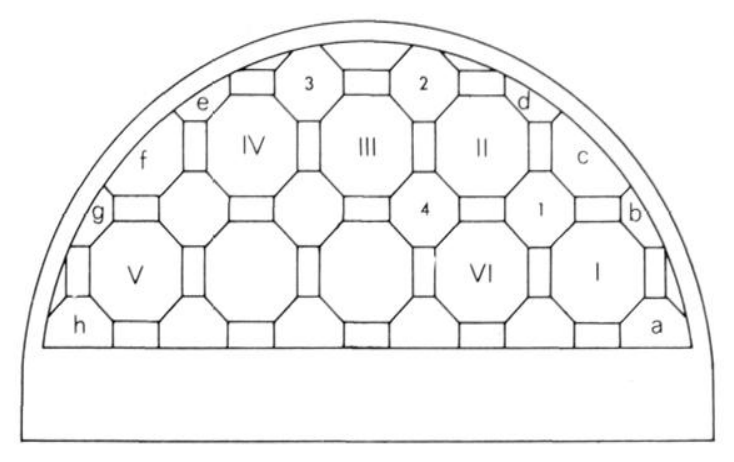

3 Schema de composition de la mosaïgure de l'abside $A$.

pièce existe et a été recherchée, parfois un peu laborieusement, par la reprise de certains thèmes décoratifs, et une cohérence interne dans l'organisation du champ des tapis. Il faut ajouter que la disparition de la mosäque sur les trois quarts de sa surface nous empêche d'avoir une idée globale des deux parties et ne nous permet pas de saisir exactement comment la grande étendue de décor géométrique s’acerdait avec le pavement à dominante végétale de l'alsside.

\section{La mosaïque de l'abside A (figr. B).}

Celle-ci se compose d'une bordure (2) ('m) a fond noir sur lequel se détache un large ruban ondé bistre et blane, dans les replis duguel alternent des ralices de lotus trifides cernés de noir, dont les deux feuilles bleu-pâle entourent un pistil fusclé jaunâtre. La composition du tapis est simple et harmonieusement équilibré : grands et petits octogones adjacents semi-réguliers déterminant des rectangles, ces trois motifs de base étant liés par une tresse à deux brins qui les enserre en un résealu relativement aćré. Cirands et petits octogones se retrouvent, mais tronqués sur une plus ou moins grande étendue selon leur position sur les bords du ranevas. On remarquera que les six grands octogones subsistant (au moins partiellement) présentent tous une bordure interne circulaire, chargée pour quatre d'entre eux, d'un rinceau de feuilles cordiformes, alors que les quatre petits octogones encore visibles en sont dépourvus. Quant aux rectangles disposés en cartourhes alternattivement horizontaux et verticaux, ils répetent un motif de cabble en arc-en-ciel (rectangles horizontaux; et un remplissage en chapelet (rectangles verticaux). Les octogones tronqués de la ligne de chute de la composition n'offrent pas de telles symétries et comportent des ornements varies. Le déer de l'absile repose done essentiellement sur un jeu entre l'octogone, le cercle et la croix (cette dernière ayant ses branches formées par les cartouches rectangulaires), l'unité étant donnée par la tresse qui court entre ces divers éléments. Nous retrouverons ces quatre composantes dams le parement de l'ecus qui fait suite à l'abside.

Les grands oclogones.

Octogone I : fond blanc, bordure interne circulaire ornementé d'un rinceau de feuilles cordiformes alternativement tite-berche, dont les deux bulbes sont noirs et la pointe verte. Les rameaux d'où naissent ces feuilles sont bruns rehaussés de noir. Jee centre du motif est un rercle à fond blanc 
frappé d'un fleuron à quat re fleurs trilides jaunes cernées de noir. séparées par des feuilles lancéolées brun rouge et noires (fig. 4 .

Octogone II : fond blinc, mème bordure intérieure que pour le précédent. Le centre est un rercle a fond noir chargé d'un norud de sialomon dont les boucles sont de couleur vert bistre, bleu pàle. Banc, brun et rose (fig. 6 ).

()ctogone III : cel octogone. en posilion centrale dans le parement. est le plus travaillé. Fond grisi. sur lequel se délache un cercle entouré d'une large bordure en couronne de laurier, dont les feuilles bistres el blanches légirement nuancées par deux rangées de tesselles de marbre bleu pâle, ressortent aver nelleté sur un fond noir oi leur ombre apparait dentelée. Le cercle central est de couleur bistre chargé d'un scilitika noir compliqué d'un effet d'ailes de moulin noires el rouges a degreses sur fond blane.

Octogone IV : fond hlanc mime bordure intérieure que dans les octogones I et II. Le cercle central. dont le contour noir est ornementé vers l'intérieur de denticules éralement noires, est à fond brun pàle et bleu tris (marbre). Le motif qui l'ornemente est une bordure d'ondes à 6 lobes cernés de lesselles blanches. le centre de cette roue étant noir frappé d'un petit cercle blanc et brun pàle (fig. ì).

()ctogrone $\mathrm{V}$ : I ris mulilé. ()n distingue néanmoins un fragment de l'octogone à fond gris chargé d'un rercle décoré d'un rinceau de feuilles rordiformes analogue à ceux des oclogones I. II et IV.

Orlogone $\mathrm{VI}$ : il n'en subsiste qu'un fragment de la bordure en feuille de laurier, identique is lil couronne de l'oclogone III.

Les petils oclogones.

Octogone 1 : sur le fond blanc s'enlive un fleuron à huit lobes qui rappelle le motif du grand orlogone IV, mais chaque lobe est séparé du suivant par un calice de lotus trifide, alternativement jaune avec pointe centrale hleue, et hleu avec pointe centrale brun pâle. Au cœur de l'octogone, deux octogones inscrits. le plus petit occupé par quatre sabliers emboîtés de couleur rose et bistre (fig. 1).

Ocloggone 2 : fleurons composé de quatre fruits (coings, cédrats?) rougreâtres, séparés par quatre calices de lolus de couleur bistre rehaussés de tesselles noires qui soulignent le mouvement évasí du calice (fig. 6).

()clogone 3 : le morceau intact montre qu'il s'agissail d'une composition d'écailles rouges, blanches et noires.

Ocloggone 4 : tris mutilé lui aussi; on distingue le début d'un carré à degrés dont la bordure étail traité: en ciible en arc-en-ciel, brun, blanc, bleuté.

Les octogones de la ligne de chute du canevas.

Octognone a : sur fond noir, onze solides évidés, groupés en deux rangées de quatre et une rangée de trois éléments. Pour donner un effet de volume a ces solides, chacune des couleurs dominantes de la rangée est soulignée par une teinte plus foncée (rose, rouge brique, jaune pâle, bistre, bleu. bistre, et le contour extérieur est rerné de blanc, tandis que l'évidement du solide est marqué par un groupe de six tesselles noires (fig. 7 ).

Oclogone b : deux peltes assez disproportionnées tète-bèche. Fond blanc, l'intérieur des peltes étant brun et bistre. l'apex central surmonté d'un pompon noir et rouge.

Octogrone a : damier d'osselets noirs et blanes (fig. 4).

Oclogrone d : fond blanc décoré de deux demi-cercles placés sur les bords de l'octogone, l'intérieur de res cercles étant marqué par une série de denticules noirs. 


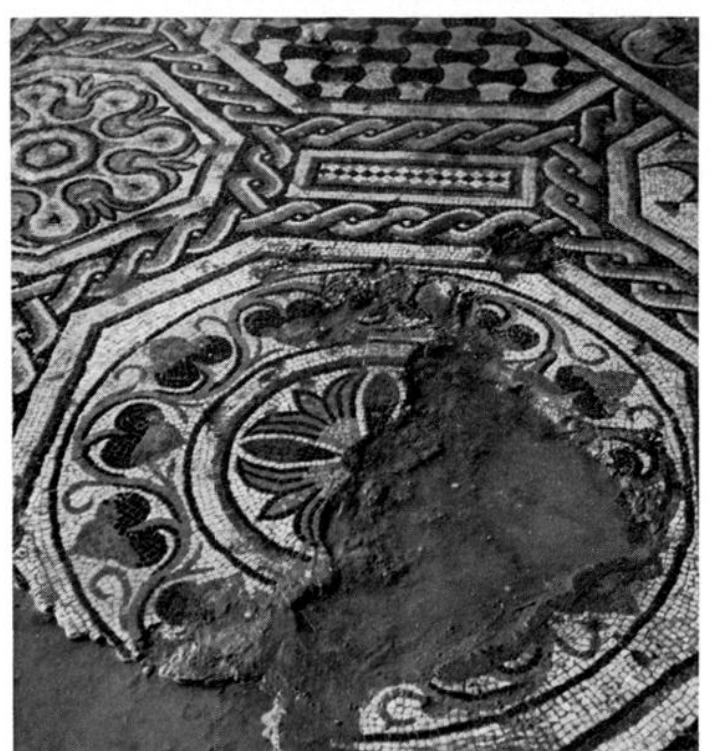

4 Octogone I.

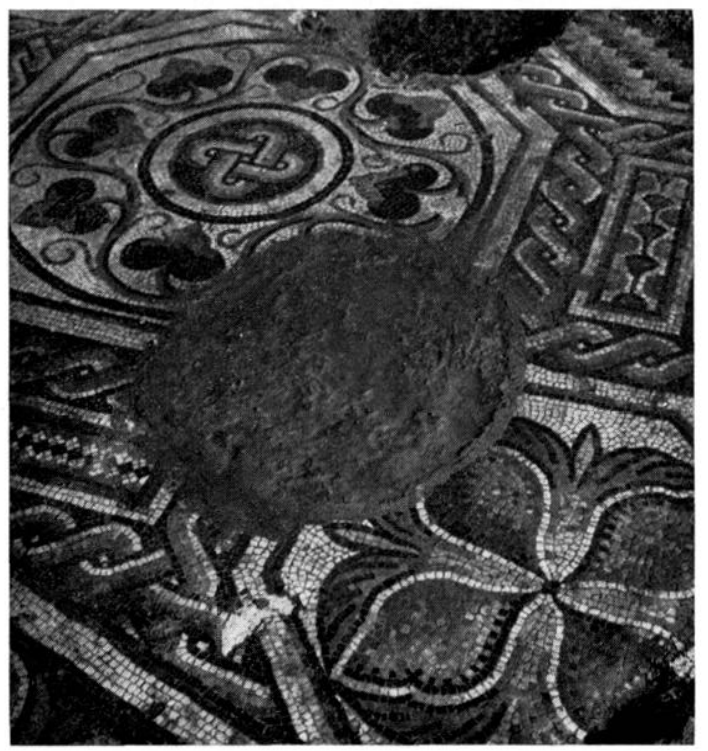

6 Oclogones II et 2.

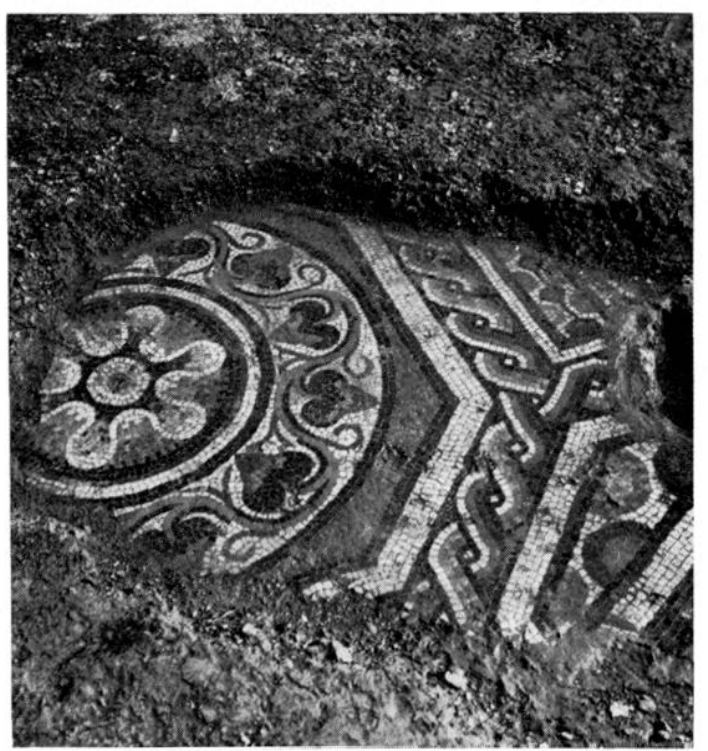

5) Oclogone IN.

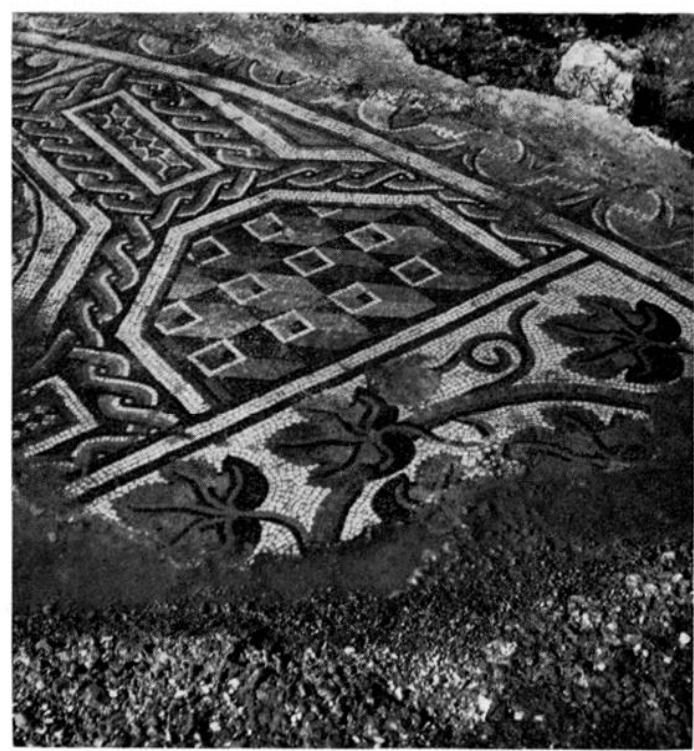

7 Oclogone a et rallonge.

Octogrone e : mème décor avec quatre demi-cercles.

Oclogone 1 : roues de peltes brunes, bleues, aver apex à pompons rouges.

Octogone g : demi-cercles comme dans d et e mais au nombre de trois (fig. 5 ).

Octogrone $h$ : une patère et l'extrémité d'une autre sur fond blanc. L'intérieur concave de l'objet est rendu par un cercle en dégradé de bleu gris et de blanc. Il est également possible d'y voir un miroir ${ }^{12}$.

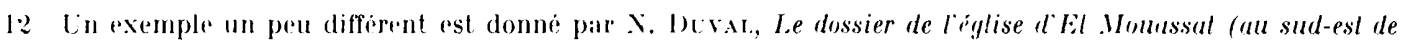
sfax, Tunisie), dans Antiquiles africaines, 8,1974 , p. 169-170, fig. 11-12. Les miroirs sont tres rarement figures seuls 
Cartouches rectangulaires : les cartouches horizontaux sont remplis par un fraugment de câble en arr-en-ciel constitué par une ligne centrale de petits carrés noirs de 4 tesselles sur la pointe, entourés de chaque coté far deux groupes de quatre tesselles bleues et blanches en damier. I.es rartouches verticaux portent une sorte de chapelet de triangles à un còté curviligne terminé par deux demi-cercles. sur les longs rotés des rectangles sont disposés des demi-cercles, symétriques les uns des autres par rapports aux éléments en chapelel firg. 6 , el 7 .

Bande de rallon!fe : elle n'est conservée que sur une faible élendue, à l'est du pavement. On y voil l'extrémilé d'un rinceau de feuilles de vigne: la ligge du sarment est bisitre et brune soulignée de noir. développée par des vrilles. I.es feuilles. neltement échancrées. sont traitées dans deux tons de vert tendre. rehaussees de tesselles noires ainsi que les nervures lig. 7 :.

Comme on le voit par eette analyse de détail. à la rigueur de la ronstruction de lensemble répond une grande varièté el mème une certaine fantaisie, dans les remplissages, notamment dans les ortogones tronqués. Ia densité des rubes est assez forte flans un carré de $10 \mathrm{~cm} \times 10 \mathrm{~cm}$, environ 140 tesselles), les joints peu apparents. On est frappé par l'harmonie des diverses tointes : un calcaire blanc, un marbre blanc; un rouge brun, un rose ; un gris tirant sur le bleu (marbre) ; un jaune pâle et un jaune tirant sur l'orange ; un vert tendre et un bistre, un noir profond. C’est avec cetle palette de dix nuances que le mosaïste a realisé son pavement dont la polychromie reste très fondue, peut-être à cause de la prédominance des teintes bistre et verte.

\section{La mosaïque de l'aecus A' (fig. 8-11).}

La réussite de l'oecus est moindre, car malgré la tresse à quatre brins qui entoure. la mosaïque, son unité fait défaut. Deux rectangles occupent les angrles norl, deux carrés les angles sud. Cette dissymétrie est accentuée par les personnages qui y sont représentés : en pied pour les rectangles A et $\mathrm{B}$, en buste pour les carrés (i et I). Le tapis géométrique central présente deux motifs assez hètérogènes. I)ans l'angle supérieur Est, on aperçoit l'extrémité d'un enroulement d'entrelacs dont une boucle est traitée en laurier (feuilles blanches et rouges sur fond noir). l'autre en lignes de carrés à degrés blancs sur fond rouge vif, la troisieme en tresse a trois brins (blanc, rouge, bistre). I ce seml coillet subsistant alu cerur de celte bourle est à fond noir frappé d'un fleuron blane à quatre fleurs lotiformes. Jeux petits carrés conservés montrent, l'un un norud de sparterie, l'autre deux peltes opposées blanches sur fond noir, dont les pointes sont terminées par des triangles à degrés. On ignore comment ce lécor pouvait se raccorder à la trame qui oceupe la majeure partie du pavement : celle-ci est ronstitué d'octogones semi-réguliers et de croix déterminant des hexagones oblongs (figr. 8). I ce lien entre ces trois éléments est à nouveau la tresse, romme dans la mosaïque de l’abside, mais l'impression générale est tout autre, car les figures de base sont plus reduites et la tresse prend une importance disproportionnée, envahit le champ et parail elouffer. en quelque sorte. les motifs du canevas. Enfin, une troisième faiblesse nuit à l'éfuilibre dre l'ensemble. I la suite d'un remaniement de l'entrée de la piece, la trame des octogones, croix et hexagones a ete reprise a galuche du seuil,

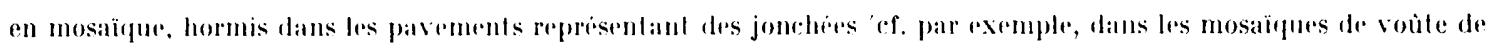
sainte-lonstance, a liome. 


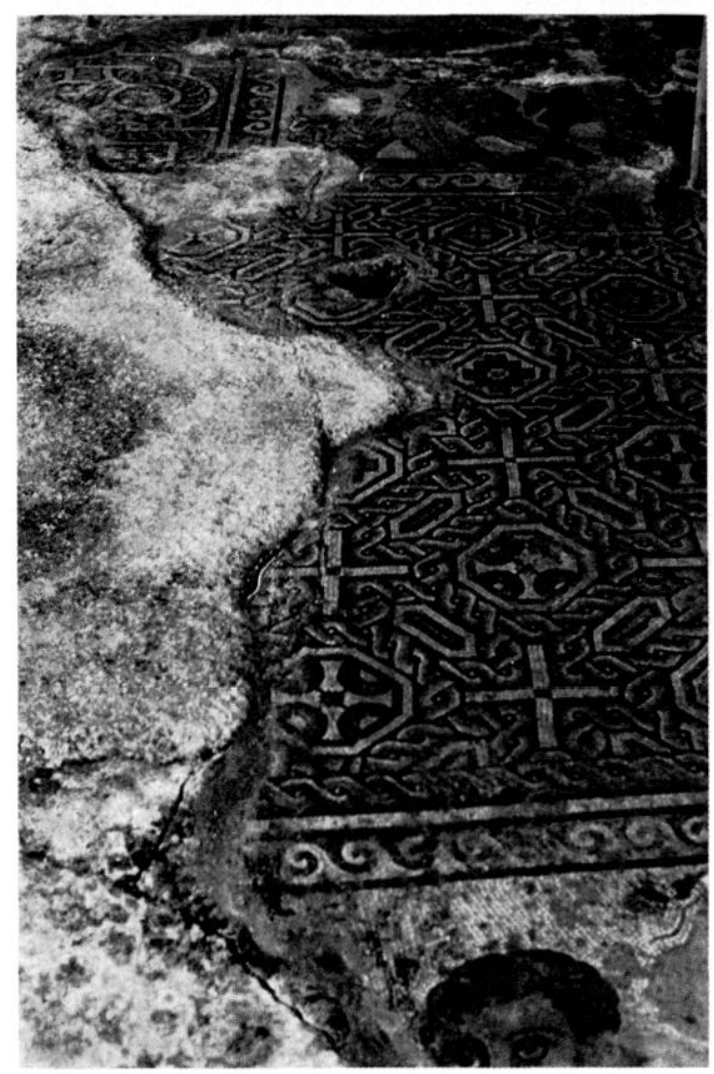

$x$ Mosaïgue de l'eecus, tapis central.

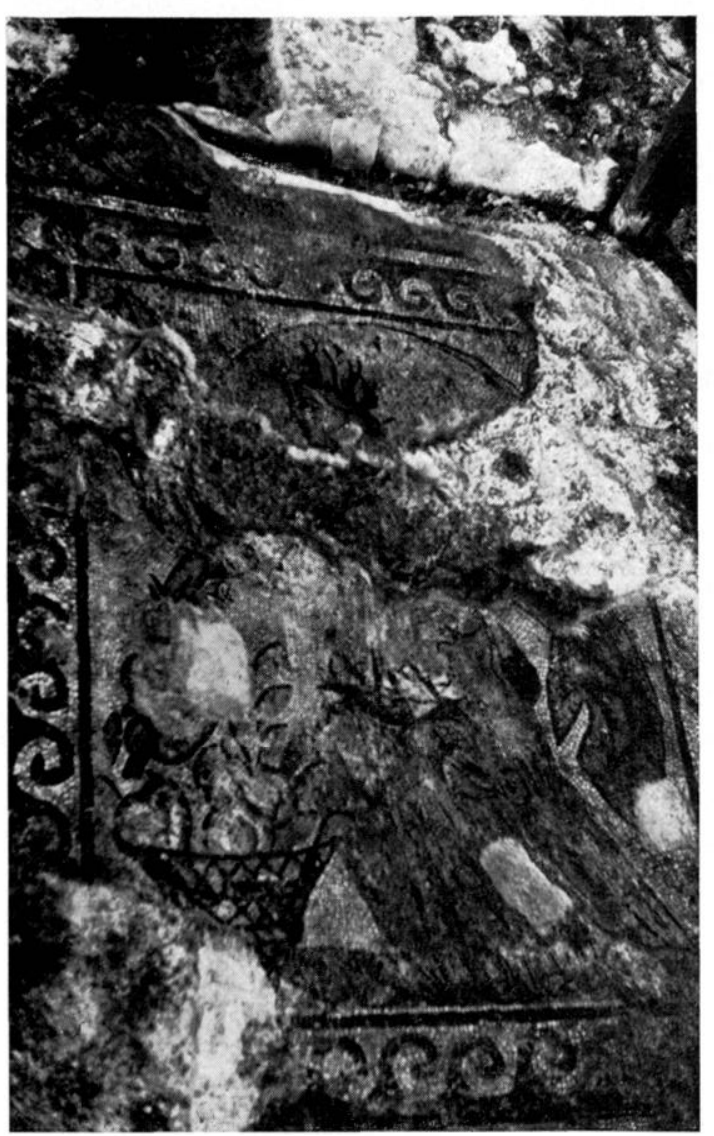

9 Panneaul 1.

mais de facon décalée, si bien que l'alignement des figures n'est plus respecté. sil'on ajoute que l'on repere en plusieurs points des incohérences de détail et des raltrapages dans les motifs de ligne de chute du tapis, on comprend que ces modifications aient emperche d'obtenir dans ce pavement la belle régularité de la composition de l'abside. Cependant, malgré son apparence à la fois monotone et surchargée, ce tapis géométrique n'est pas sans intérêt pour ses motifs de remplissage. Les croix sont blanches, frappées a l'intersection des branches d'un carré de 9 tesselles rouges, ce détail disparaissant dans la partie remaniée. près du seuil. Les octogones sont tous chargés d'une croix traitée differemment dans chaque alignement diagonal. T'antôt c'est une croix noire qui divise un carré surr la pointr. rouge et blane, tantôt clle est frappée d'un cercle blanc et se surimpose à un carré de couleur bistre. A trois reprises, elle utilise un des remplissages des octogones tronqués de la mosaïrpue de l'abside, en déterminant entre ses branches curvilignes quatre demi-cereles opposés. bordés intérieurement de denticules noirs. Dans la partie gaurle du pavement. flanquant le panneau C, les octogones subsistants sont différents puisque le fond est noir chargé d'un noud de Salomon blane et gris, ou d'un coussinet dont le centre est materialise par une croix rouge. La tresse elle-mème, qui serpente rntre les figures géométrique's varie légèrement : à droite du panneau C, les torsades des brins sont assez étirées, alternativement rouges/blanches, et bistres/blanches sur fond noir, tandis que dans la partir la mieux 


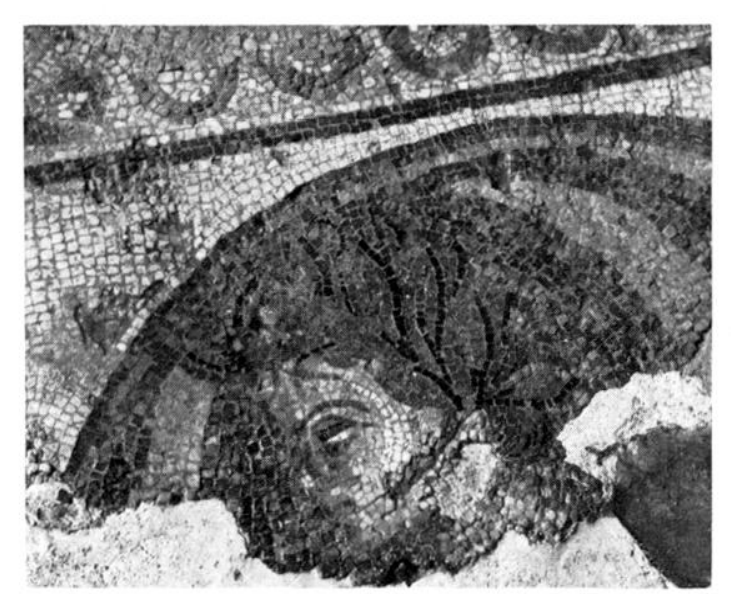

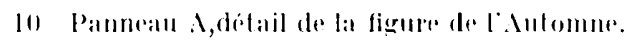

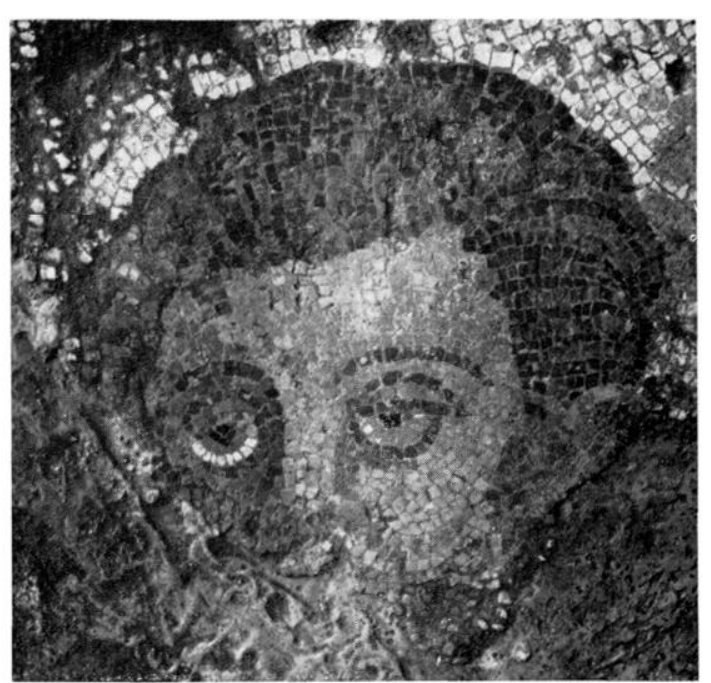

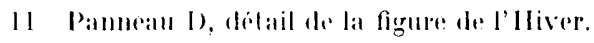

conservé (entre les pannealux A et I)), les boucles sont plus serrées, les couleurs moins heurtés. Tous res indices attestent un remaniement indéniable, dì à une main moins exercé pour la zone inférieure du pavement. On retrouve cette différence entre deux mains dans la facture des quatre panneaux qui cantonnent la mosiäque.

Pinneau $\perp$ (lig. 9 et 10 : dans un carlre rectangulaire $11.37 \mathrm{~m} \times 1,16 \mathrm{~m}$ ) doublé intérieurement par une bordure de postes rouges sur fond blane dont l'enroulement a une forme triss aplatie, apparaît un personnage féminin. en pied, vu de profil el comme saisi en marche, dans un mouvement rapide vers la gauche du spectateur. La têle est couronnée de feuillages (laurier?j verts dont les rameaux se détachent en noir. La chair du visage est rose avec des rehauts de blanc, qui dominent l'ovale de la joue, l'wil cerné de noir, le front et le menton ì peine soulignés de lesselles plus rougres. Un ample manteau bleu et vert s'enfle derriere elle aver la forme arrondie du voile tradilionnel de la nympha relificans. Ce voile qui lui entoure les spaules (partie triss dégradée s'envole en un pan qui monte vers l'angle galuche du lableall. el retombe en arriere dans l'angle inférieur droit. le personnage est revitu d'une lunique rose serrée par un pli plus sombre a la ceinlure. qui recouvre une longue robe rose descendant jusqu'aux pieds; les plis du lissu sont rendus par des files de tesselles verticales rouges, le bas se termine par une bordure marquée de lignes de cubes noirs. De la main droite. elle tient un canard vert par les palles arriere. de la gauche, un liovre gris et rose dont la fourrure est striée de cubes noirs sur l'échine. Devant elle. sur la grauche. est placé un panier d'osier tressé rempli de gros fruits jaunes a bulbes. entremilés de feuillage vert, dans lesquels on peut probablement reconnail re des cédrats ou des citrons ${ }^{13}$. Celte figure est mieux venue que relle qui lui fait pendant. par l'élégance de la silhouetle el la tinesse de la polychromie. On y relive douze teint es. dont trois en paite de verre bleu profond. Wea tirant sur le vert émeraude. vert Véronisej. des marbres roses, blane's, jaunes orangrés. un jaune vif, un rouge brique, deux ralcaires blanes el noirs, les couleurs dominantes élant le bleu et le vert du voile. Iensili des cubes : jusqu'a $160 / 170$ : $14 \mathrm{~d} \mathrm{dm}^{2}$.

l'anneau li : placé dans l'angle supérieur gaturhe et entouré de la meme bordure de postes rouges. il est malheureusement tris mulile $(1,01 \mathrm{~m}$ >07.7). Fïpure féminine enveloppée dans un voile

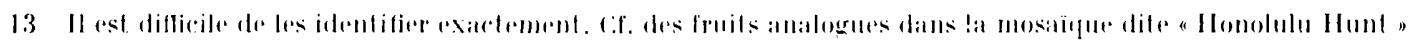

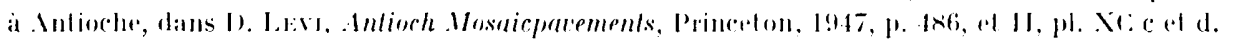


qui a le même mouvement circulaire que précédemment, mais le personnage est de face, et tient dans la main droite une longue guirlande de roses et de feuilles. un buste.

Panneau $C:$ : disparu pour la plus grande partie, sauf un pan de manteau (?) rouge qui recouvre

Panneau D (fig. 11) : seul le haul du lableau est conservé. Visage masculin barbu dont la chevelure, ramenée sur le devant du front, est soigneusement crantée. Les yeux sont largement ouverts, fixes, sans expression. Sur sa droite, un objet à panse bosselée, terminé par une extrémité en croc, est suspendu par un lien accroché à un clou noir de grande taille. On pourrait penser à une besace, mais nous proposons plutòt d'y voir une de ces citrouilles bien connues des pays méridionaux. L'exécution du portrait est très inférieure, les teintes sont moins variées (blanc, rose, orange, bistre, vert noir) et la facture plus lourde.

Comment faut-il identifier ces figures? Seul le panneau B n'est pas douteux : le personnage féminin qui tient à la main une guirlande de roses est le Printemps. Le panneau Ci est trop abìmé pour y reconnaitre la saison traitée. On est tenté de voir dans le panneau A une représentation de l'Automne, comme nous y convient le lièvre, le canard et le panier de fruits. Dans ce cas, le panneau I) serait l'Hiver. La première difficulté tient à ce que l'orlre traditionnel de succession des saisons n'est pas respecté puisque l'Hiver et l'Automne sont intervertis. D'autre part, les images de l'Hiver avec comme attributs les canards et le lièvre sont bien attestées : c'est le cas de la mosaïque des Mois à Thysdrus ${ }^{14}$, de celle d'Aumale ${ }^{15}$, de celle de Beit-Jebrin ${ }^{16}$ ou encore des sculptures que porte le coffret de Fenek (Musée de Budlapest) ${ }^{17}$. Troisième difficulté, le melon, l'aubergine, ou la courge sont généralement les légumes qui accompagnent les mois d'été comme, par exemple, sur la mosaïque de Kabr-Hiram ${ }^{18}$ ou sur celle de Tégéée ${ }^{19}$. Enfin, à Thysdrus dans la Domus Sollertiana ${ }^{20}$, une autre mosaïque des Saisons montre une figure féminine couronnée de fleurs et portant un lièvre dans laquelle on ne peut voir que le Printemps. C'est dire combien cette iconographie reste flottante. Pourtant, une découverte récente vient confirmer l'identification du panneau A avec l'Automne. Sur un pavement de Sidon ${ }^{21}$, aux abords du temple d'Echmoun, les quatre saisons sont disposées comme à Loupian, aux angles d'un tapis à décor géométrique, les Saisons des angles supérieurs étant dessinées en pied, celles des coins inférieurs en buste. De plus, le carton suivi pour l'Automne est presque identique : même jeune femme drapée, même attitude légèrement fléchie sur la jambe gauche, même attribut, le lièvre. On aurait donc à Loupian, comme dans la mosaïque du Liban, une composition en chiasme, disposition qu'il est possible d'expliquer par des remaniements dans l'ordonnance de la pièce, mais qui peut tout aussi

14 I.. Fotciner, Decotuvertes archéologiques à Thystrus en 1961, Tunis, 1961, p. 45, fig. 17, 01 pl. XXXI1.

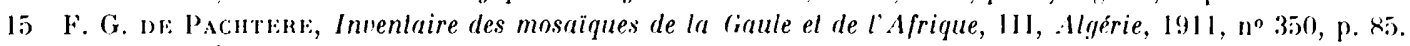

16 H. Sterx, Éludes sur le calendrier de 3.5, Paris, 1953, p. 23\%. Inate proposé : ves.

17 (․ .1. A. Haxpmax, The season sarcophagus in Jumbarlon (Jaks, Cambridge, 1951, 11, pl. $145, \mathrm{n}^{\circ} 362$.

18 H. Stren, ibid., p. 261.

19 H. STrers, ibid., p. 22:, 261, 263. Dinte: début du ves.

20 I. Focchi: op. cil., pl. XXVI.

21 Pavement découvert en 1971, encore inédit. Nous devons ce renseignement a .I me J. Christophe. L'analogie vaut seulement pour la ressemblance des cartons utilisés, car la facture de la mosaïgue libanaiso indifue une date plus haute (ive s. 
bien se comprendre comme un procédé artistique, que seul notre goùt moderne pour les séquences rationnelles envisage moins facilement ${ }^{22}$.

La conclusion qui s'impose en considérant d'un point de vue global ces deux pavements est leur bonne qualité d'exécution, l'aspect encore très classique de la facture, surtout pour la partie absidée de cette pièce d'apparat. L'éturle de détail fait apparaitre des morceaux particulièrement soignés (la figure de l'Automne) et des maladresses dans l'adaptation des canevas géométriques. Vais il ne faut pas oublier que nous regardons ces mosaïques sur des relevés d'ensemble, alors que l'étendue de la pièce, et la différence de niveau qui sépare les deux parties soulignée par la colonnadei devaient masquer les faiblesses de l'unité du décor.

\section{Datalion.}

\section{L'abside $A$.}

L'aspect très aquitain frappe dès l'abord, non pas tant par la grille géométrique cmployée ${ }^{23}$ (elle est peu répandue en Gaule et dans les provinces) que par les motifs de remplissage et leur traitement. D'une manière générale, on remarque le soin apporté à remplir tous les vides résiduels du tapis. Le fond n'apparait pratiquement pas, tant les motifs sont enserrés par le chaînage de la tresse, et tous les espaces qui se prêteraient mal à un remplissage cohérent sont pourvus d'éléments décoratifs assez maladroitement disposés, comme c'est le cas pour ces curieux demi-cercles qui mangent, en quelque sorte, la surface déjà très réduite des octogones tronqués de la ligne de chute du canevas. Outre cette aversion pour le fond blane (qui n'est d'ailleurs pas l'apanage exclusif de l'Aquitaine à époque tardive), on note un emploi caractérisé des motifs à base d'ares de cercles (écailles, osselets, peltes, éléments en chapelet des cartouches), le seul motif simple à base de carré étant le damier, employé très parcimonieusement. On retrouve rette tendance dans des pavements tardifs comme ceux de Taron ${ }^{24}$ et Lescar ${ }^{25}$ (Pyrénées-Atlantiques), de SaintSeverer6 (Landes), Séviac ${ }^{27}$ (Gers), généralement datés de la fin du we siècle ou du début du ve siècle. Ln autre point de rapprorhement avec l'Aquitaine est l'emploi de la couronne de laurier. Presque inconnu en Ciaulere, ce motif fréquent en Afrique, est éqalement très

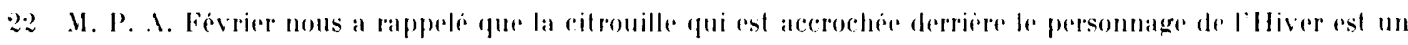
altribut parfaitement adapte a cette saison dans un contexle grograptique meriblonal. Ea bffet, de nos jours encore, une des plus grandes fêtes hivermales du pays niegois n'est-elle pas precisément la lête des cougrourdins durant layuelle on a coutume de promener les plus geosses "comgourdes" courges an provengal avant de fes decorer ed de lessuspendere dans les habitalions?

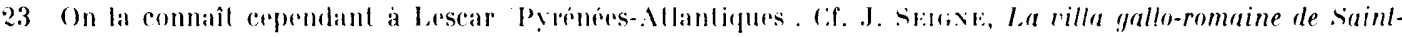

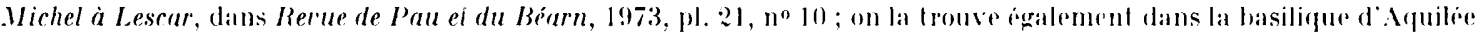

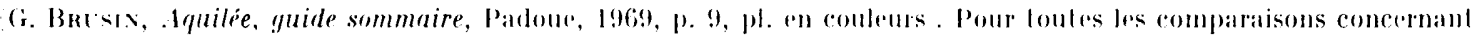

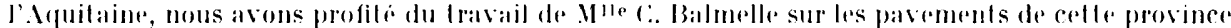

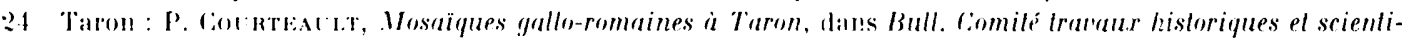
fiques, sechion archénlogie, 1909!, p. 117-1:21, pl. XI

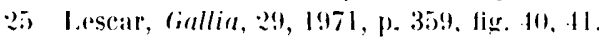

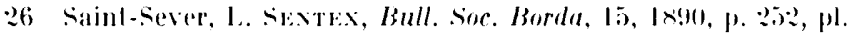

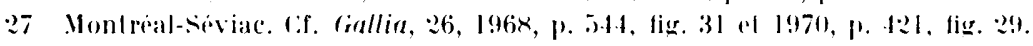

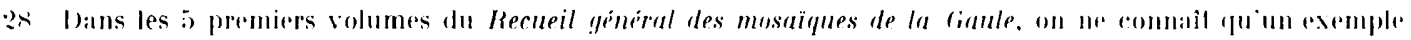
approchanl, a Blanz-les-Fismes Province de Belgigue, mais dont H. Stern sondigne lorigine ef be caractere probahle- 
répandu en Aquitaine. Enfin, quelques détails précis se retrouvent de manière particulièrement frappante dans les pavements de Séviar : le rinceau de feuilles de lierre bichrome dont la base est à double bulbe, les feuilles de vigne largement echancrées, et surtout un motif rare, les demi-cercles garnis intérieurement de denticules noires29. Quant aux fleurons, ils existent à Séviac comme à Lescar. On rapprochera, enfin, les pampres de la rallonge de ceux de Sarbazanon. C'est au fond tout Ir décor végétal dans son ensemble qui semble ètre influené par les mosiäques tardives de l'Aquitaine.

\section{l'ipctes il'.}

Le schema principal (octogones, hexagones et croix) est connu a ballze (Gers) ${ }^{31}$ et à Lalonquette ${ }^{32}$. mais traité de façon différente de Loupian, dans la mesure où les trois éléments composant la grille restent très proportionnés. I)ans le cas présent, la prolifération de la tresse aux depens des motifs donne à cette mosaïque un raractère beaucoup plus oriental. I). Levi a analysé l'évolution de re canevas dont la tète de série est donnée

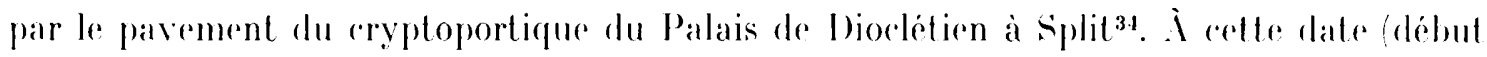
du $w^{\mathrm{e}}$ sièce), lo ranevas est encore très striet, ronstitue par la seule juxtaposition rles élements noirs sur fond blane. Il se eomplique rapidement par une ornementation varjépe a l'intérieur des ortogones at des hexagones, tandis que les croix restent très linéaires at sans remplissage. Céest le cas, par exemple dans la mosiäque des Vents de la villa de Frampton (I)orsed) datée entre 315 et 350. où l'on voit déja apparaître la tresse qui antoure les octogrones, mais dans une composition plus aréée qu'à Louphian. La complieation des remplissages se fait déjà plus nette dans la mosiäque des bains dits de Pompeianus prese de Oued-Athmenia ${ }^{36}$ que loon peut dater de la deuxieme moitié du IV siècle. On peut voir cette tendance se préciser dans un pavement d'Aquilée ${ }^{37}$ de la fin du re sierle, mais cerst a Antiorhers que la grille prend sal forme la plus surehargée,

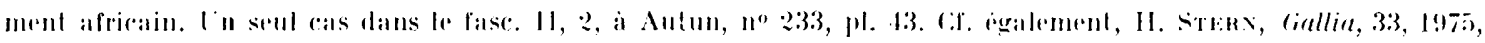
1. I $16 x$.

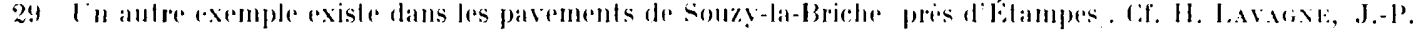

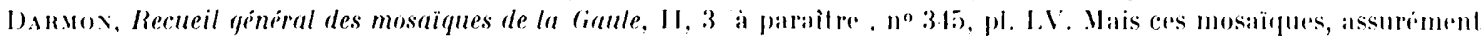
lardives ve, ves. occupent une place tout a fait à parl dans la produrlion de la lyomatise.

30 sarbazan Iandes, fiallia, 25, 1967, p. 364, fig. 5x.

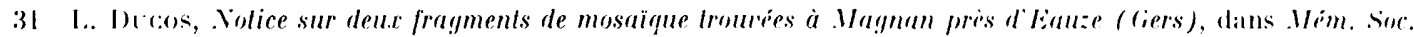
arch. Midi de la France, 1, 1 $\times x \cdot 2 \times \times 3$, 1). 371-373, fig. 1.

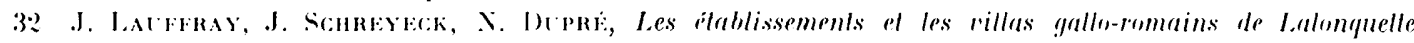

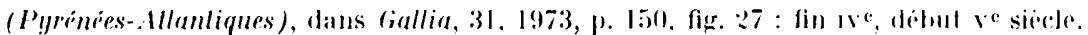

33 I). 1.ki, op. cil., p. 113 , fig. 156 ed note 1.

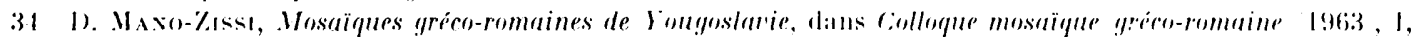
196i), p. 2990, fig. 11 .

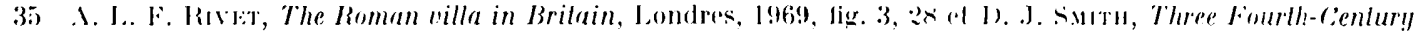
schools of mosaic in Roman Brilain, Colloque mosaïque greco-romaine, $I$, p. IIII.

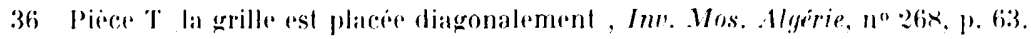

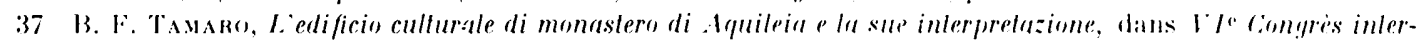

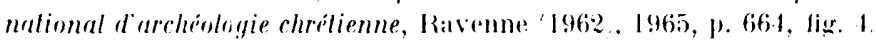

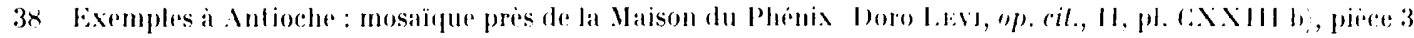

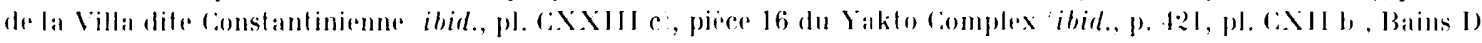

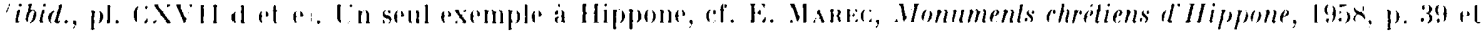

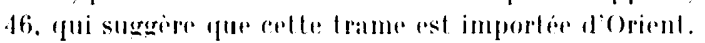


au re siècle : non seulement octogones et hexagones portent des motifs divers, mais les croix elles-mèmes sont ormementés et cernés par la présence envahissante de la tresse. Ce schéma restera en vogure au moins jusqu'a la fin du ve sièrle, comme on le voit en Gèrece ${ }^{39}$. La forme qu’il revèt a Loupian. entere l'austérité linéaire des premiers exemples et la richesse decorative des parements d'Antiorhe. nous parait devoir ètre placéer vers le début dur ve siecte.

Si l'on examine non plus la l rame, mais les figures, d'autres remarques appuient cette datation. Le panneatu de lolliver possede bien des traits de visages de facture tardive: frontalite, absener de proportions, disparition du fond pour insister sur l'objet significatif

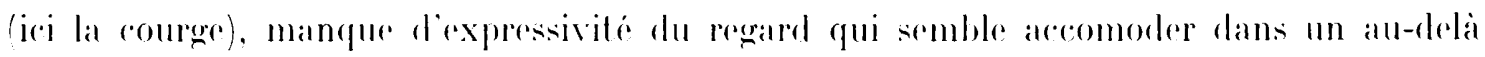
lointain, et mème, la roiffure en calotte ramené sur un front bass, antant d'éléments qui nous rapprochent davantage du ve siecle que du re ta figure de l'Automne, moins datable en apparenee pare qu'elle dérive d'un rarton, appartient à la mème époque par l'absence de rendu du volume et des differents plans dans l'espace. On le remarque notamment dans le dessin du corps du lievere : un simple pointille de tesselles noires marque l'échine

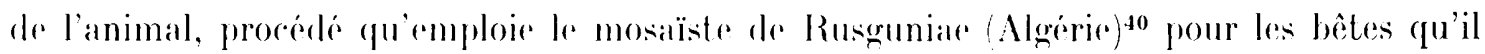
représente dans son parement.

Enfin, un motif extrèmement rare, ne paraît pas avoir existé avant lat première moitié du ve siècle. Il s'agit de ces rhapelets de triangles à un côté curviligne contenu dans les cartouches verticaux. L'unique comparaison qui peut ètre etablie doit ètre cherché dans Jes mosä̈ques de la basilique de Misis (Mopsueslia) en Cilicie où l'aspect figuré de l'objet est encore visible : un trépied de métal dont la colonnette ouvragée est faite d'une sucesssion de cabochons triangulaires et de perles ciselées, et supporte, au sommet. une lampe à huile. ì Loupian, par exces de stylisation, le motif a perdu tout rapport aver la réalité concrede at est devenu purement décoratif. Il se rapproche par la d'un type d'ornement apparente : la bande geminée où alternent cabochons et pertes, dont la vogue est particulierement grande a partir du début du ve sierdet2.

Les divers exemples auxquels nous avons fait apper pour replacer cette mosiäque dans son contexte stylistique nous ont fait passere de l'Aquitaine a l'Afrique et a l'orient du début du ve siècle. Cóst à ce mème cheminement que va nous entrainer l'étude de la mosä̈que de la pièce li.

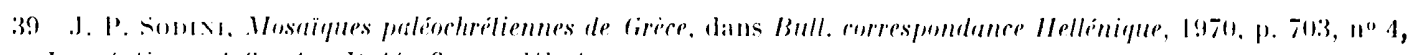

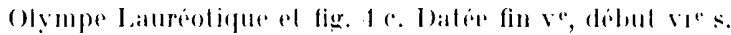

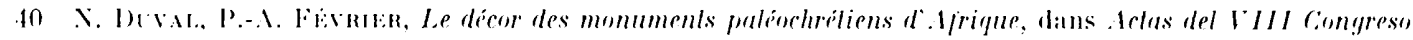
Int. Ant. Cristiana, Barerlone, 19669 1972, pl. IX.

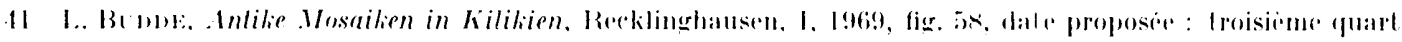

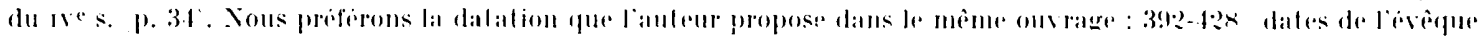

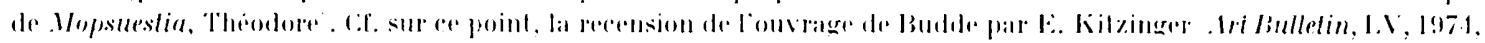
p. 1.11 qui penche plutiot pour le se sirele.

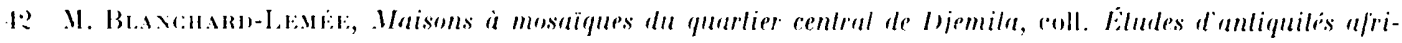
caines, Palris, 1975, p. 118-119. 
LE PAVEMENT DE LA PIÈCE E (fig. 12)

Cette grande pièce $(10,55 \mathrm{~m} \times 8.85 \mathrm{~m})$ montre un pavement harmonieusement composé, où un thème architectural se lie à un décor végétal. Lne large bordure $(1,18 \mathrm{~m})$ représentant un portique, court autour d'une composition géométrique constituée par un grand nocud d'entrelacs dont les quatre boucles en l'se prolongent sur deux axes orthogonaux.

L'ensemble n'est conservé que sur à peine un quart de la surface, mais les éléments encore en place permettent d'en restituer les lignes directrices. Le portique est constitué de petits piliers trapus, aux chapiteaux stylisés supportant des arcatures très surbaissées, les angles etant formés d'ares en demi-cercles qui soulignent le caractère de projection géométrale sans effet de perspective qu'il faut attribuer à ce thème iconographique ${ }^{43}$.

I)ix arcatures et dix pilastres sont partiellement conservés sur un total de trente-deux. Le fùt, des colonnettes et leurs chapiteaux sont chargés de motifs variés dont la répartition n'est pas ordonnée selon une fréquence fixe, tandis que les décors qui occupent les entrecolonnements se succèdent dans un ordre $a b / a b$ respecté uniformément, ce qui donne un certain rythme au déroulement des arrades sans engendrer la monotonie.

Les remplissages qui ornent les fûts des colonnes sont au nombre de quatre el appartiennent au répertoire traditionnel de la mosaïque, sans rappeler en rien l'architecture proprement dite : petit damier à cases noires, blanches et grises, grille diagonale à handes blanches séparées par une ligne de tesselles noires, bande de chevrons blancs et brun pâle, ou blancs et gris, guillochis simple, de couleur brun, bleu el blanc. I.es chapiteaux sont tous à décor végétal très stylisé oì l'on peut reconnaître une prédominance de feuilles lancéolées (laurier?) blanches ou grises cernées de noir sur fond brun. Sur deux d'entre eux seulement a été esquissé un effort pour rendre la profondeur en opposant le fond rouge brun de la partie médiane du chapiteau aux cótés en cubes gris. Quelques feuillages sont échancrés, et rehaussés de filets jaunes. Du sommet de chaque chapiteau sort une vrille à volute.

Entre les arcatures alternent deux types de figures. Tantolt un vase vu en perspective où sont représentés l'ouverture. la panse et le fond sur le mème plan, tantòt un groupe formé par deux cornes d'abondance encadrant un objet divers (vase, palire ou miroir), surmonlé par une coquille. Les deux vases les mieux conservés sont légèrement différents (fig. 13) : l'un est rendu dans les gris. la lumiere sur les flanc's étant marquée par des lesselles blanches, mais l'artiste ayant voulu égralement représenter le fond avec le mîme efiet de volume a dessiné sur le còté, une forme ellipsö̈dale en pàte de verre rougre. Le second est comme vu de dessus aver une encolure intérieure rendue en petits rubes bistres, landis que le fond, également vu en perspective, montre le mème effort pour rendre le volume du vase par le dessin d'une ellipse rouge sur un fond de pâte de verre de couleur verte. Le pied du vase, triss stylisé. est entierement en pàle de verre verte. Ie ces deux vases s'échappent, de maigres rinceaux de vigne, sans feuilles, oi les grappes de raisin sont formées de trois petites baies rouges frappées d'un point blanc, qui terminent des liges jaunes rehaussées de noir. La stylisation de ce décor n'a d'égal que son còté extrêmement moderne qui fait penser aux recherches des peintres cubistes. L'autre type de figures est plus classique : une roquille occupe le rul-de-four. avec l'attache de la valve placée en haut (sous forme d'un triangle rouge; . tandis que les cannelures internes du coquillage sont tracées en pointillé noir sur fond gris et bleu. De chaque colé, deux

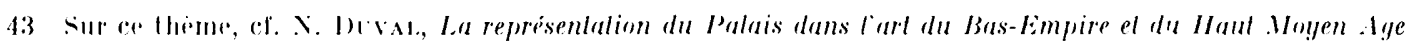
dapres le psanlier delrechl, dans Cahiers archéologiques, 15, 1965, p. 20x-2:4. 


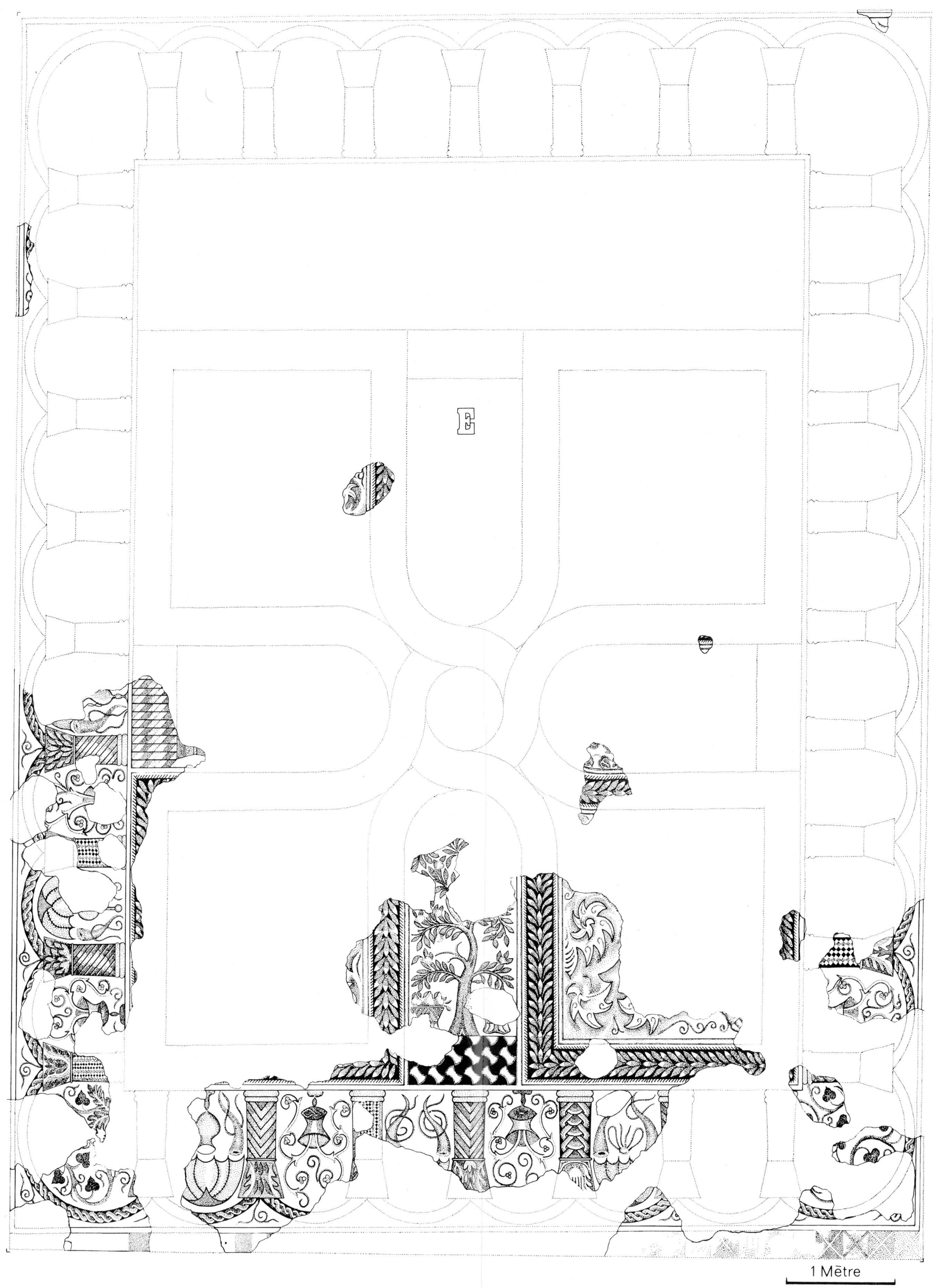





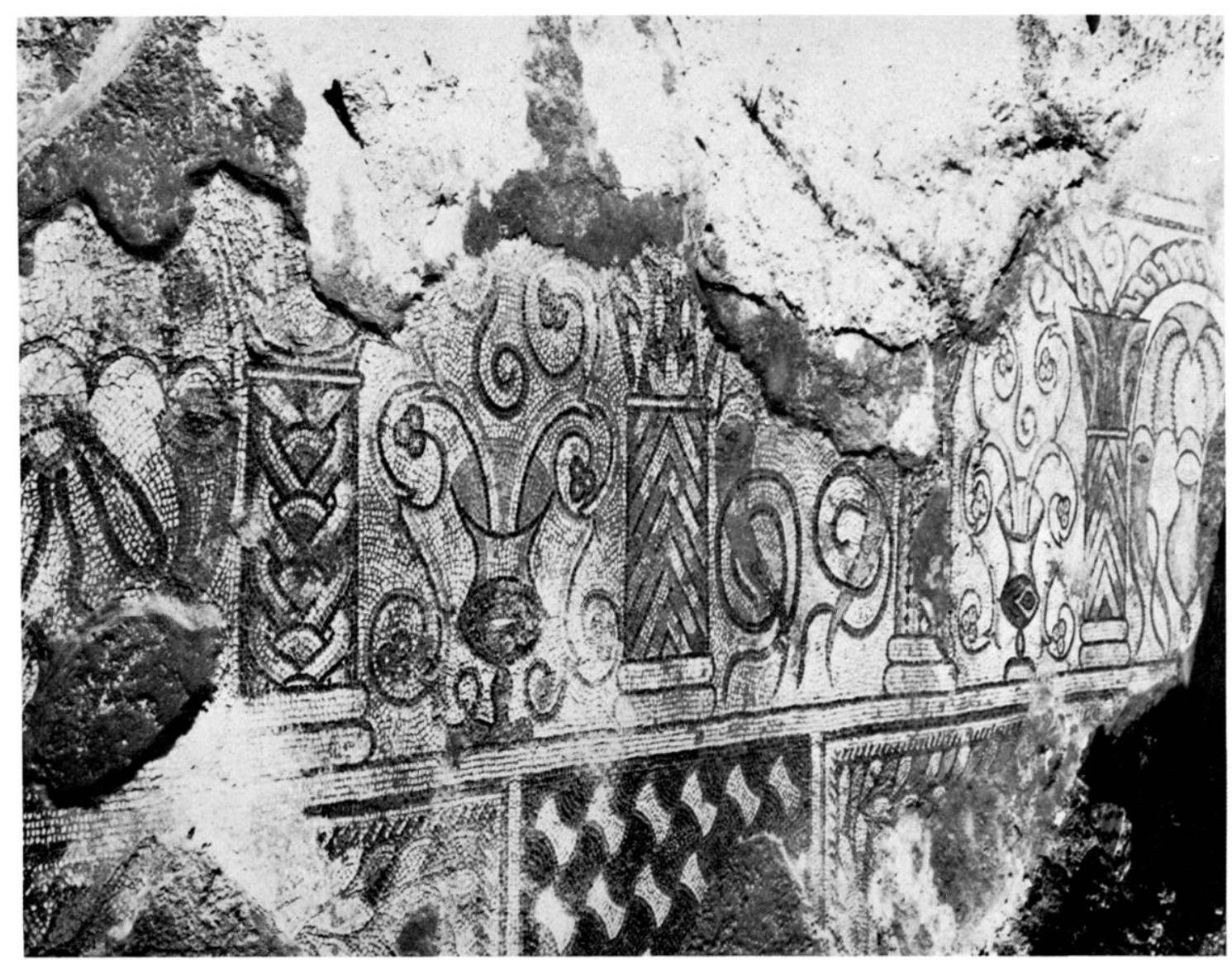

13 Parement F, deslail du portique.

cornes bistre se font pendant, entourées a leurs extrémilés par des fouets rouges et noirs aux circonvolutions plus ou moins contournées. Entre les cornes, se place, tantòt un vase élancé de couleur jaunâtre, tantòt une patère traitée de la mème façon que dans la mosaïque de l'abside A, tantòt un simple noud avec des flots de rubans retombants.

Les entrecolonnements des angles de la bordure montrent un rinceau de feuillages verts terminés par des feuilles cordiformes a deux bulbes noirs. La lresse qui couronne tous les cintres est bleue, rouge el blanche.

L'impression d'ensemble est double : une grande rigueur donnée par la trame architecturale, l'aspect massif des piliers, le caractire répétitif des molifs, l'uniformité créée par le lien de la tresse, el d'autre part, le còté composite des remplissages et le pittoresque des notalions de détail.

Le décor du lapis, pour le peu qu'il en reste, est d'une grrande régularité. Les quatre larges boucles en $\mathrm{U}$ forment un noud circulaire au centre. et déterminent quatre carrés. Dans les boucles, court une guirlande de laurier ininterrompue, dont les feuilles blanches el jaunes cernées de noir se détachent sur un fond noir, finement dentelé sur les deux bords extérieurs. La partie interne de l'une des boucles en $\mathrm{C}$ est presque intacle. (On y voit une rallonge rectangulaire à la partie inférieure, portant un damier d'osselets hancs et noirs entoures d'une file de lesselles rouges. Sur ce socle, s'élève un figuier au trone gris d'où partent des branches porteuses de rameaux aux feuilles allongées gris vert, parmi lesquelles on aperçoit quelques liques rouges. symétriques de chaque còté de l'arbre. deux paniers, (dont le mieux ronservé a la panse ornée d'un damier à bandes diagonales noires, rouges, blanches) sont remplis de figues, trois d'entre elles dépassant du bord; l'autre panier est tries endommagé. 
Des carres qui cantonnaient le tapis. nous n'avons qu un mince temoignage : fe bas d'un cratere a godrons place dans un angle. doil sorl, en posilion diagonale, un rinceau d'acanlhes anx larges échancrures bistres cernés de noir. entre lespuelles se dressenl des hampes donl les rosses a volules sont de couleur rouge.

A premiere vue le slyle de celte mosaingue paraît plus lardif que celui du parement IA'. Toulefois, on note que certains motifs de la mosaïque de l'abside sont fidolement repris : les osselets. la bordure en guirlande de laurier. les damiers qui remplissent les fùts des colonnelles. el juspua a la patire cou le miroir objet assez rare dans le répertoire de la mosiöque pour qu'il ne soil pas sams avoir valeur de rappel. L'étude des schémas de composilion el des motifs ornementaux fait d'ailleurs apparailre que les deux ensembles appartiennenl sinon au mème alelier du moins à la mème période.

Comme pour les mosä̈ques de la piere à abside, les rapprochements qui sömposent

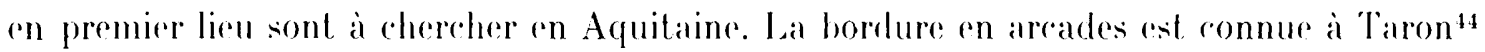
(1) à Seviac4s où un petit fragment conservé permet de reconnaìtre le sommet de l'areature ot l'extremite de la coquille qui remplissait le coul-de-four. L a arbres qui oecupent les boucles en l'sont présents également à Séviac of à Taron et les fruits qui sont disposes dans les paniers sont, comme a Loupian. certainement des figales. I seviace enfin. figurent les colnes

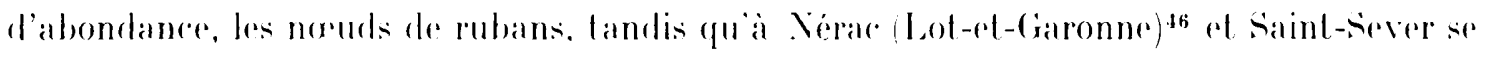

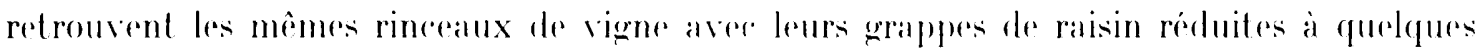
petites baies rouges. Enfin, les vases en position diagonale plarés dans les angles du lapis sont bien attestés en Aquitaine : a Sarbazann ol a Valentine (I Iaute-(iaronne) ${ }^{48}$ où pour

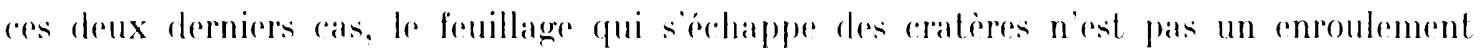
d'aranthe, mais une pyramide fruillue. Tous aes motifs sont rombines dans un fragment. de mosäque de Seviaret9 qui résume an quelque sorte le parrement de loupian : dans une boucle en L', une requille d'ousont une pramide veregetale sur un fond d'aranthes dentelees, portant an centre des cedrats, des grappes de raisin al une feuille de vigne.

On ne manquera pas non plus de penser aux seupturis des sareophages de l'éole d'Aquitaine ou ranthares, pyamides feuillues, rinceaux de vigne à larges fenilles constituent les écements traditionnels de leiconographie (fig. 14, sareophage de Montlouis). Malheureusement. la datation de ces sarrephages est encore contestee rel ne pent servir de critere pour nos parementson, mais d'autres comparaisons tirés de regions exterieures à l'Aquitaine permettent de proposer une ehronologie plus précise.

La bordure en arcades est illustrése par de nombrem parrments tant en Gialue que

11 Voir mote: 24.

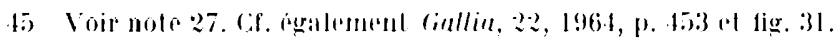

16 Giallia, :29, 1971, p. 355is, figr. 25) a 37.

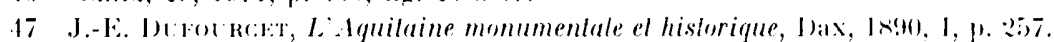

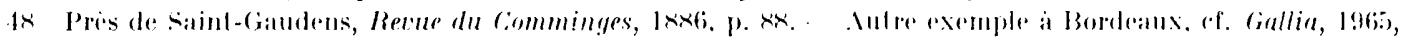
p. 417 , lig.

49 Acturlement conserve au Muser de lectoure. Pour un exemple dementement d'acanthes dentelés a siviac, of. Ciallia, 22, 1964, p. 453, fig. 30.

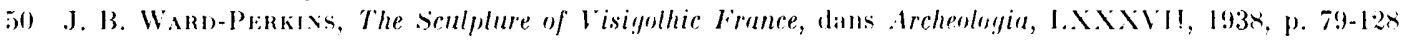
pour une datalion hatute. I). Fussans, La chronologfe des sarcophages d Aquilaine, dans Acles du le Congres inler-

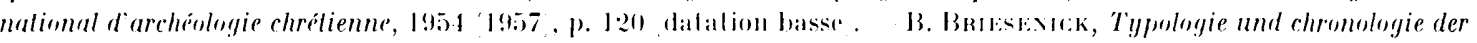
$\therefore$ II. Gallischen sarliophaye, Mayence, 196:2, passim. 


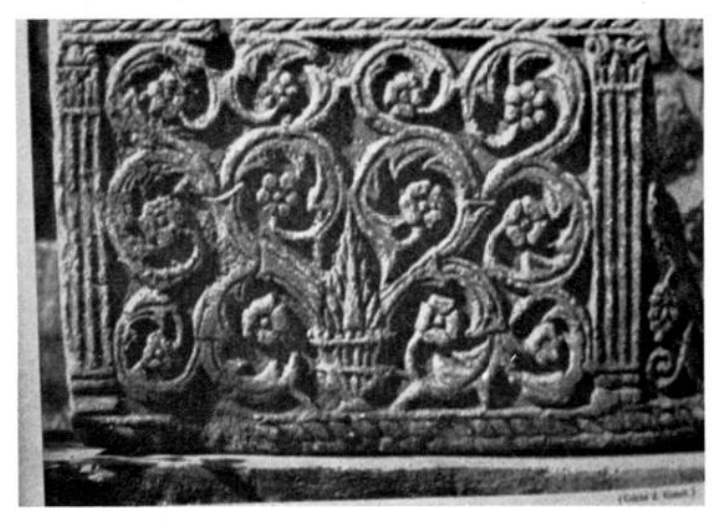

11 sircoplage de Montlouis.

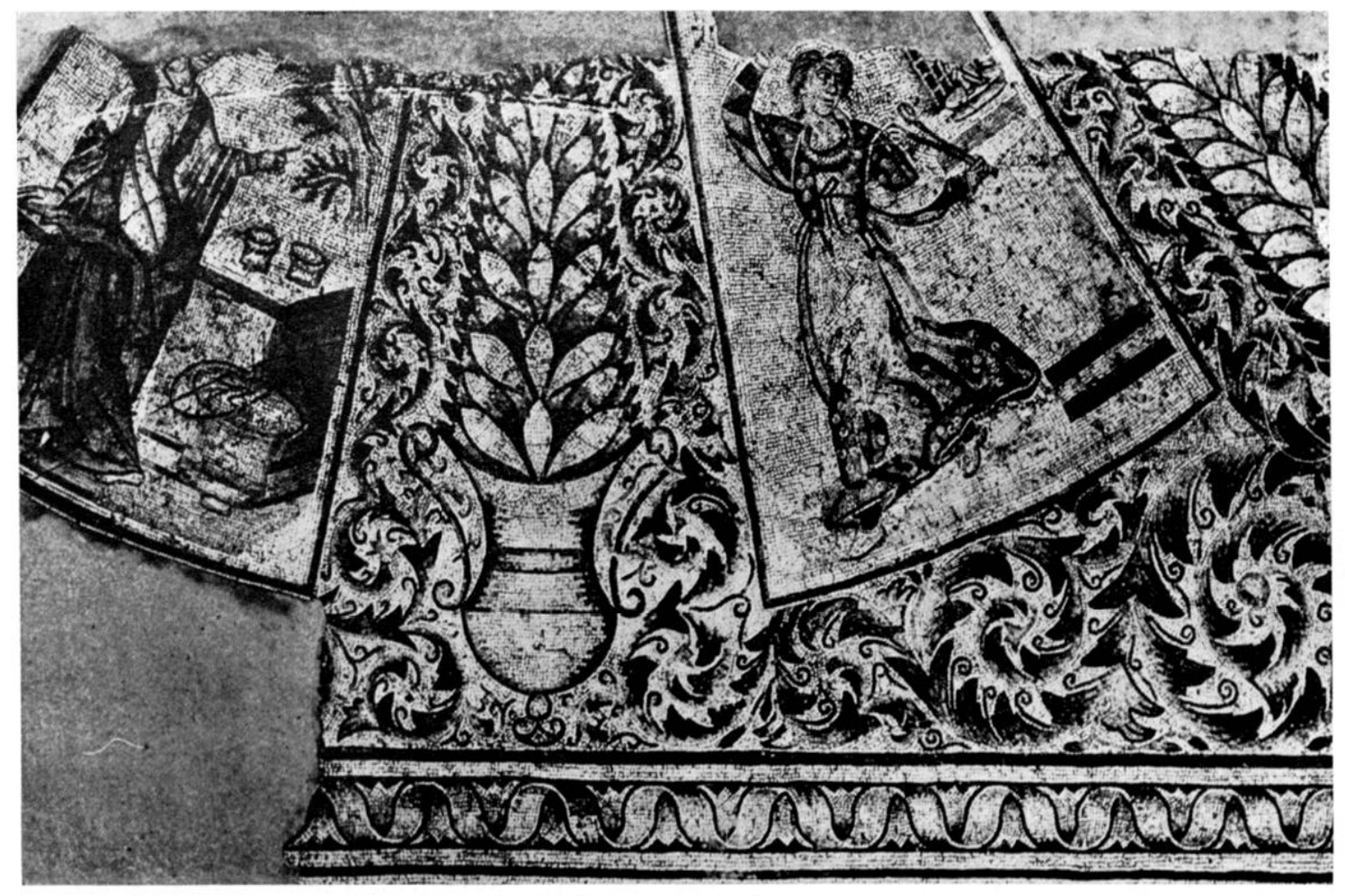

dans les autres provinces, mais le plus souvent sous une forme grèle et linéaire, si stylisée que la référence originelle à un véritable portique architertural a disparura ${ }^{51}$.

Ce décor ne reprend valeur de projection d'architedure an sol qu'a partir du re siecle et se trouve bien illustré par les pavements africains du ve siède : dans la mosä̈que de la contre-abside de Reparatus (datée de t7i) a Al-Asnam (ex-Orléansville) s2, dans le

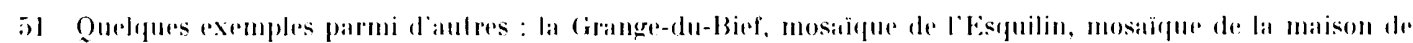

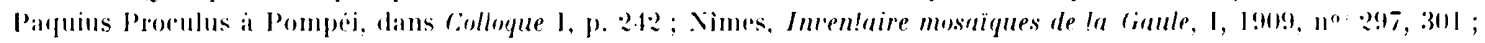
l.anurium, Archeologia classica. 17, 1965, pl. XXIX.

52 X. Inval, P.-A. Jismis, op. cil., pl. VI, fig. $x$. 
pavement de la basilique de la Skhira $\left(\mathrm{vi}^{\mathrm{e}} \text { siècle }\right)^{53}$, sur une mosaïque du British Museum d'origine africaine ${ }^{54}$; mais on la repère également en Grèce ${ }^{55}$ et en Espagne ${ }^{56}$ aux ve et $\mathrm{vl}^{\mathrm{e}}$ siècles.

Quant au système d'entrelacs à boucles en $\mathrm{U}$ orthogonales qui décore le tapis, il est ignoré en Gaule, mais semble avoir connu une réelle faveur au Proche-Orient et en Grèce. J.-(h. Balt $y^{57}$ en a donné trois exemples du we siècle, en Apamène, dans l'église d'Jerbet Mūqa, à Qumhane près de Lama, et à Apamée même dans la salle $B$ de l'édifice dit au Triclinos. Il existe aussi dans la chapelle de la Vierge au Vont Nebo ${ }^{58}$ au ve siècle, et dans la basilique paléochrétienne de Jelphes ${ }^{59}$ ( $\mathrm{v}^{\mathrm{e}}-\mathrm{vJ}^{\mathrm{e}}$ siècle?).

Ln autre point de repère iconographique est fourni par le motif des canthares placés dans les angles. Là encore, outre les exemples aquitains déjà cités, on rapprochera d'une mosaïque des Baléares ${ }^{60}$ de deux pavements de Carthage ${ }^{61}$ (fig. 15), d'un autre agrémenté de coquilles à Thuburbo Majus ${ }^{62}$ ou encore de la mosaïque de la basilique IV de TebessaKhalia ${ }^{63}$. Ia fourchette chronologique fournie par tous ces exemples laisse la possibilité d'opter pour le pavement E de Loupian entre la fin du $\mathrm{IV}^{\mathrm{e}}$ et le début du vi ${ }^{\mathrm{e}}$ siècle. Mais d'autres analogies de détails font plutôt pencher pour le ve. Ce sont notamment les rinceaux de vigne où le raisin est composé de petits grains juxtaposés qui ont un parallèle exact dans telle sculpture de fenestella du Musée de T'ébessa ${ }^{64}$, et les vases stylisés du pilier de Kenchela ${ }^{65}$ où l'on roit la même forme ellipsö̈dale de la panse sur un pied aussi réduit qu'à Loupian. Ce type de vase est d'ailleurs des plus répandus dans l'iconographie chrétienne et funéraire du ve siècle.

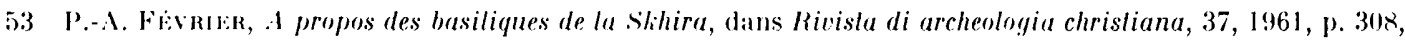
fig. 2. . . Duval, Ieux basiliques chréliennes de Tunisie meridionale, dans Cahiers archeologiques, XIII, 1962, p. 2801-281.

54 H. Strinx, op. cil., pl. XI.III, 1 a 3, qui la date du we s.

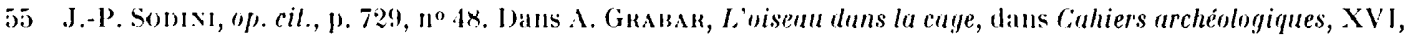
p. 10, fig. 1, elle est dater du vie s. Selon P. Isimitrion Eph. Arch. 1938-19.44, p. 42-48, du début du ve s.

56 A. Ba.ri., Las escuclas musivarias del conventus Tarraconensis, Colloque mosaïque I, p. 33, fig. I3 mosaïque dite de Vitalis i. Le motif existe done bien aussi en milieu profane. C'est te cas notamment d'un pavement d'une villa de Puppot en Tunisie indit signalée par X. Duval op. cil., p. 21) et dans une mosaïque de Cherchel où l'arcade devient une sorte de treille.

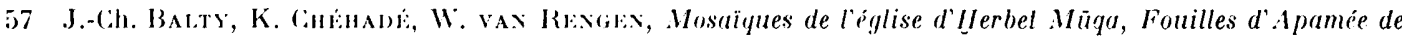

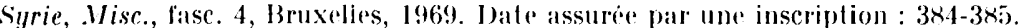

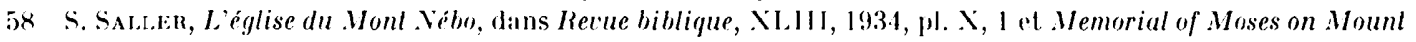
Vebo, II, Jerusalem, 1941, pl. 109, 2.

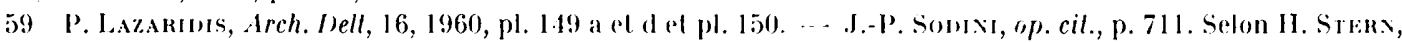
dans Cahiers archéologiques, 15, 1965, p. 35, note 45, ce pavement serait plutot du vie s. ; mais M. Cimatzidakis, Propylaën zur Byzantinischen Kunst, p. 237, pl. 176, propose le ves.

60 P. DE PaLol, En lorno a la iconografia de los mostaicos cristianos de las islas Baleares, dans Sancho el Subio, $\mathrm{X}, 1966, \mathrm{p}, 36, \mathrm{vi}^{\mathrm{e}} \mathrm{s}$.

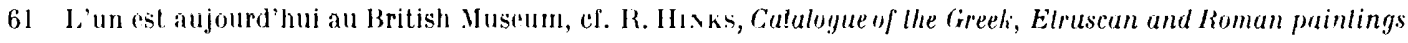
and mosaics in the B. M., londres, 1933, p. $\times 1-96, n^{\circ} 29$, at-11; l'autre est au Musee du Bardo Bull. Comile travaux

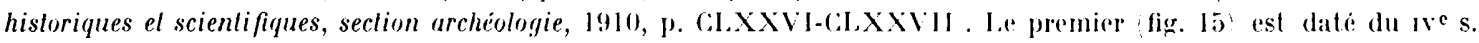

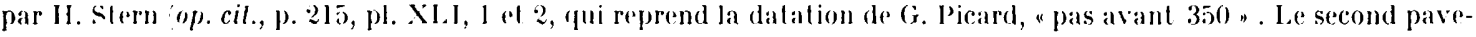
ment est de facture plus tardive, ve, peut-être même dỏbut du vies.

62 G. Picars, La mosaĭque romaine en Afrique du Yord, dans Gazelle des Bealux-drls, 1958, p. 190, fig. 12. $\mathrm{v}^{\mathrm{s}} \mathrm{s}$.

63 J.-P. Botcher, Le lemple rond de Tébessu-Khalia, dans Iibyca, V, 1956, p. 15, fig. 9. La basilique IV est datée de la fin du $\mathrm{v}^{\mathrm{e}}$ siecle.

64 X. Deval, P.-A. Févrter, op. cil., pl. XVII, fig. 34. On les rapprocherait volontiers des grappes de la colonne de la Daurade a Toulouse (Musée du Louvre, cf. l). Fossars, op. cit., p. 324, fig. 5, daté du vo s.

$65 \quad$ N. Ulval, P.-A. Févaler, op. cil., pl. XXI, fig. 42. 
Tous ces exemples pourraient être encore multipliés, comme on est toujours tenté de le faire pour des ceurres tardives. $\mathrm{A}$ partir de la deuxième moitié du ive siècle, comme on l'a souvent répété ${ }^{66}$, la désintégration des formules iconographiques traditionnelles entraine la création d'une véritable Koinè de motifs dans laquelle puisent tous les artistes du monde méditerranéen. Le grand nombre d'exemples que nous avons relevés en Afrique du nord ou au Proche-Orient témoigne de ce caractère composite et de cette circulation des cartons (et peut-être même des ateliers) qui sont utilisés indifféremment dans plusieurs techniques à la même époque. Càr non seulement le trésor commun des canevas et des remplissages passe d'un bord à l'autre de la Véditerranée, mais il est aussi transporté sur tous les supports, marbres sculptés de sarcophages, orfèvrerie, mobilier d'église, mosaïques d'édifices religieux ou profanes, stucs ${ }^{67}$, etc.

D'un point de vue moins général, les mosaïques de Loupian présentent un autre intérêt, car elles montrent pour la première fois l'influence des ateliers aquitains en pleine province de Narbonnaise. Loupian, rappelons-le, est aux confins du territoire de la cité de Nìmes ${ }^{68}$, et c'est pourtant la région du Gers, des Landes et des Pyrénées-Atlantiques qui fournit les comparaisons les plus proches. On imagine assez bien qu'en ce début du $v^{e}$ siècle, le riche propriétaire de la villa se soit tourné vers les maîtres de l'école d'Aquitaine, dont l'abondance, la richesse d'invention et la nouveauté devaient séduire davantage que la production artistique provençale, encore très classique, italianisante de style, et finalement peu renouvelée depuis l'essor des ateliers de la vallée du Rhône.

Henri Lavagne et Richard Prudhomme.

66 H. STrks, passim.

67 Par ex. les stues du palais Omayade de Khirbat Al Mafjar. (if. C. Corswri., Early mustim architeclure, Oxford, 1969, pl. 99 el 100 f.

68 D. Cinvin, Le lerriloire de Béziers dans l'Anliquilé, op. cit., p. 225 el carte no 13. 